\title{
Modification of materials to decrease of quantity of radiation defects in an implanted-heterojunction rectifier
}

\author{
E. L. Pankratov • E. A. Bulaeva
}

Received: 3 February 2012/Accepted: 7 May 2012/Published online: 24 June 2012

(c) The Author(s) 2012. This article is published with open access at Springerlink.com

\begin{abstract}
It has been recently shown that manufacturing of an implanted-junction rectifier in a semiconductor heterostructure for optimal relationship between energy of implanted ions, materials and thicknesses of layers of the heterostructure $(\mathrm{H})$ after annealing of radiation defects gives us possibility to increase sharpness of $\mathrm{p}-\mathrm{n}$-junction and at the same time to increasing of homogeneity of dopant distribution in the doped area (Pankratov, Phys Lett A 372(11):1897, 2008; Proc SPIE 7521:75211D, 2010a, b). In this paper we consider a possibility to decrease quantity of radiation defects, which were generated during ion implantation, using porous epitaxial layers of the heterostructure.
\end{abstract}

Keywords Implanted-junction rectifier .

Decreasing of quantity of radiation defects .

Heterostructure with porous layers

\section{Introduction}

In the present time intensive refinement of elements of integrated circuits (IS) occurs. One of the most refined elements is $\mathrm{p}-\mathrm{n}$-junctions and their systems (bipolar transistors and thyristors) (Lachin and Savelov 2001; Grebene 1983, Gotra 1991). It is attracted an interest increasing

E. L. Pankratov $(\bowtie) \cdot$ E. A. Bulaeva

Nizhny Novgorod State University of Architecture and Civil Engineering, 65 Il'insky Street,

Nizhny Novgorod 603950, Russia

e-mail: elp2004@mail.ru; kisa12111991@yandex.ru

E. L. Pankratov

Nizhny Novgorod Institute of Commerce,

27 Lenin Avenue, Nizhny Novgorod 603950, Russia sharpness of $\mathrm{p}-\mathrm{n}$-junctions, and at the same time increasing homogeneity of dopant distribution in enriched by the doped area. To increase the sharpness of $\mathrm{p}-\mathrm{n}$-junction, laser (Bykov et al. 2003) or microwave (Ong et al. 2006) types of annealing of dopant (for diffusion-junction rectifiers) or radiation defects (for implanted-junction rectifiers) and inhomogenous distribution of defects in doped sample or heterostructure $(\mathrm{H})$ could be used. In the works of Pankratov (2008a, 2010a, b) we consider an alternative approach to increase sharpness of implanted-junction rectifiers and at the same time to increase homogeneity of dopant distribution in doped area. Framework the approach we consider implantation of dopant in the epitaxial layer (EL) of a $\mathrm{H}$, which consist of the EL and a substrate (S). The $\mathrm{H}$ is presented in Fig. 1. Type of conductivity in $\mathrm{S}(\mathrm{p}$ or $\mathrm{n}$ ) is known. The implanted dopant produced the second type of conductivity in EL ( $n$ or $p$ ). During annealing of radiation defects, spreading of dopant distribution could be obtained. If energy of ions, thickness of EL and materials of $\mathrm{H}$ are chosen optimally, dopant due to diffusion achieves interface between layers of $\mathrm{H}$. Due to the achievement one could obtain increasing of sharpness of $\mathrm{p}-\mathrm{n}$-junction and at the same time one could obtain increasing of homogeneity of dopant distribution in doped area (Pankratov 2008a, 2010a, b). In this paper we consider porous EL. Porosity of EL gives us possibility to increase sharpness of $p-n$-junction and homogeneity of dopant distribution in doped area (Pankratov 2011). Main aim of the present paper is the analysis of influence of porosity of EL on quantity of radiation defects in $\mathrm{H}$. Accompanying aim of the present paper is the development of mathematical approach for modeling of modification of porosity during annealing of radiation defects, because the mathematical approach that has been used in Pankratov (2011), gives us possibility to describe only final stage of modification of porosity. 


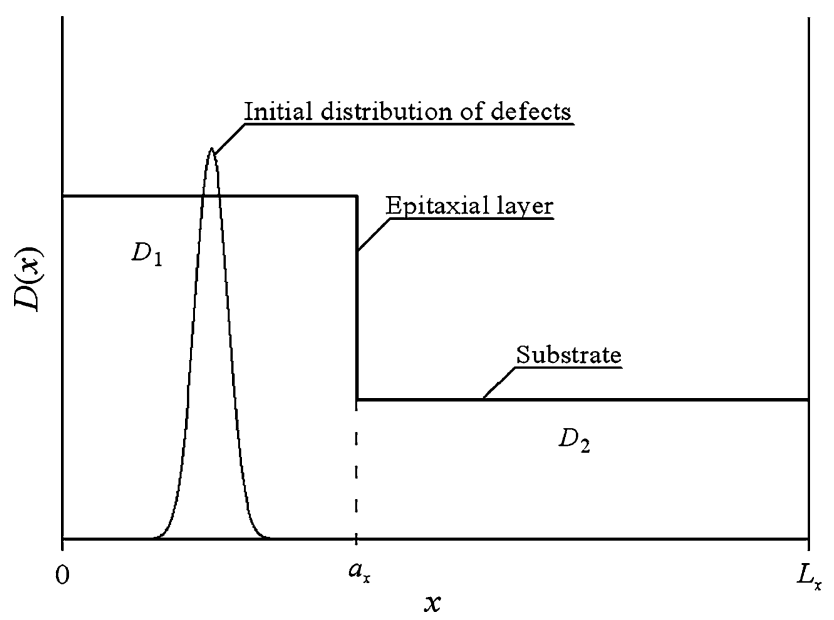

Fig. 1 Heterostructure with the epitaxial layer $\left(x \in\left[0, a_{x}\right]\right.$ and diffusion coefficient $\left.D_{1}\right)$ and the substrate $\left(x \in\left[a_{x}, L_{x}\right]\right.$ and diffusion coefficient $\mathrm{D}_{2}$ ). The figure also illustrates initial (before starting of annealing) distribution of radiation defects

\section{Method of solution}

To solve our aims we determine spatiotemporal distribution of dopant concentration. The distribution has been determined by solving the second Fick's law (Lachin and Savelov 2001; Grebene 1983; Gotra 1991; Pankratov 2011; Kitayama et al. 2000a, b)

$$
\begin{aligned}
& \frac{\partial C(x, y, z, t)}{\partial t}=\frac{\partial}{\partial x}\left[D_{\mathrm{C}} \frac{\partial C(x, y, z, t)}{\partial x}\right] \\
& +\frac{\partial}{\partial y}\left[D_{\mathrm{C}} \frac{\partial C(x, y, z, t)}{\partial y}\right]+\frac{\partial}{\partial z}\left[D_{\mathrm{C}} \frac{\partial C(x, y, z, t)}{\partial z}\right] \\
& +\frac{\partial}{\partial x}\left[\frac{D_{\mathrm{CS}}}{\bar{V} k T} \frac{\partial \mu(x, y, z, t)}{\partial x}\right]+\frac{\partial}{\partial y}\left[\frac{D_{\mathrm{CS}}}{\bar{V} k T} \frac{\partial(x, y, z, t)}{\partial y}\right] \\
& +\frac{\partial}{\partial z}\left[\frac{D_{\mathrm{CS}}}{\bar{V} k T} \frac{\partial \mu(x, y, z, t)}{\partial z}\right]
\end{aligned}
$$

with boundary and initial conditions

$$
\begin{aligned}
& \left.\frac{\partial C(x, y, z, t)}{\partial x}\right|_{x=0}=0,\left.\quad \frac{\partial C(x, y, z, t)}{\partial x}\right|_{x=L_{x}}=0, \\
& \left.\frac{\partial C(x, y, z, t)}{\partial y}\right|_{y=0}=0,\left.\quad \frac{\partial C(x, y, z, t)}{\partial y}\right|_{x=L_{y}}=0, \\
& \left.\frac{\partial C(x, y, z, t)}{\partial z}\right|_{z=0}=0,\left.\quad \frac{\partial C(x, y, z, t)}{\partial z}\right|_{x=L_{z}}=0, \\
& C(x, y, z, 0)=f_{C}(x, y, z) .
\end{aligned}
$$

Here $C(x, y, z, t)$ is the spatiotemporal distribution of dopant concentration; $k$ is the Boltzmann constant; $T$ is the annealing temperature; $\mu(x, y, z, t)$ is the chemical potential; $\bar{V}$ is the molar volume; $D_{\mathrm{C}}$ and $D_{\mathrm{CS}}$ are the coefficients of volumetric and surface dopant diffusion. Value of dopant diffusion coefficients depends on properties of materials of layers in $\mathrm{H}$, on rate of heating and cooling of $\mathrm{H}$ and on spatiotemporal distribution of the dopant concentration. It has been shown in Gotra (1991) that in high-doped materials interaction between dopant atoms and point defects increases. If the point defects have non-zero charge $\gamma e$ with $e$ an elementary charge, then the interaction leads to concentrational dependence of the diffusion coefficient. Parameter $\gamma$ depends on the properties of materials of $\mathrm{H}$ and could be integer usually in the interval $\gamma \in[1,3]$ Gotra (1991). The parameter could be larger, than 3, but probability of the case $\gamma>3$ is substantially smaller, than probability of the case $\gamma \in[1,3]$. Concentrational dependence of diffusion coefficient could be approximated by the following functions (Gotra 1991)

$$
\begin{aligned}
& D_{\mathrm{C}}=\tilde{D}_{\mathrm{L}}(x, T) \beta(x, y, z, t)\left[1+\xi_{V} \frac{C^{\gamma}(x, y, z, t)}{P^{\gamma}(x, T)}\right] \\
& D_{\mathrm{CS}}=\tilde{D}_{\mathrm{LS}}(x, T) \beta(x, y, z, t)\left[1+\xi_{\mathrm{S}} \frac{C^{\gamma}(x, y, z, t)}{P^{\gamma}(x, T)}\right] .
\end{aligned}
$$

Here $P(x, T)$ is the limit of solubility of dopant in $\mathrm{H} ; \quad D_{\mathrm{L}}(x, T)=\tilde{D}_{\mathrm{L}}(x, T) \beta(x, y, z, t)$ and $D_{\mathrm{LS}} \quad(x, T)=$ $\tilde{D}_{\mathrm{LS}}(x, T) \beta(x, y, z, t)$ are the diffusion coefficients for low-level of doping; $V(x, y, z, t)$ is the spatiotemporal distribution of concentration of vacancies; $\beta(x, y, z, t)=$ $1+\left[\varsigma_{1} V(x, y, z, t) / V^{*}\right]+\left[\varsigma_{2} V^{2}(x, y, z, t) /\left(V^{*}\right)^{2}\right]$. Spatiotemporal distributions of point defects (both vacancies and interstitials) we determine by solving the following system of equations (Pankratov 2011; Kitayama et al. 2000a, b; Fahey et al. 1989; Zorin et al. 1975)

$$
\begin{aligned}
& \frac{\partial \rho(x, y, z, t)}{\partial t}=\operatorname{div}\left\{D_{\rho}(x, T) \operatorname{grad}[\rho(x, y, z, t)]\right\} \\
& +\operatorname{div}\left\{\frac{D_{\rho S}(x, T)}{\bar{V} k T} \operatorname{grad}[\mu(x, y, z, t)]\right\} \\
& -k_{I, V}(x, T) I(x, y, z, t) V(x, y, z, t) \\
& -k_{\rho, \rho}(x, T) \rho^{2}(x, y, z, t)
\end{aligned}
$$

with boundary and initial conditions

$$
\begin{aligned}
& \left.\frac{\partial \rho(x, y, z, t)}{\partial x}\right|_{x=0}=0,\left.\quad \frac{\partial \rho(x, y, z, t)}{\partial x}\right|_{x=L_{x}}=0, \\
& \left.\frac{\partial \rho(x, y, z, t)}{\partial y}\right|_{y=0}=0,\left.\quad \frac{\partial \rho(x, y, z, t)}{\partial y}\right|_{y=L_{y}}=0, \\
& \left.\frac{\partial \rho(x, y, z, t)}{\partial z}\right|_{z=0}=0,\left.\quad \frac{\partial \rho(x, y, z, t)}{\partial z}\right|_{z=L_{z}}=0, \\
& \left.\frac{\partial I(x, y, z, t)}{\partial x}\right|_{x=x_{1}}=0,\left.\quad \frac{\partial I(x, y, z, t)}{\partial y}\right|_{y=y_{1}}=0, \\
& \left.\frac{\partial I(x, y, z, t)}{\partial z}\right|_{z=z_{1}}=0, \quad V\left(x_{1}+V_{n} t, y_{1}+V_{n} t, z_{1}+V_{n} t, t\right) \\
& =V^{*}\left(1+\frac{2 \ell \omega}{k T \sqrt{x_{1}^{2}+y_{1}^{2}+z_{1}^{2}}}\right), \rho(x, y, z, 0)=f_{\rho}(x, y, z) .
\end{aligned}
$$


Here $\rho=I, V ; I(x, y, z, t)$ is the spatiotemporal distribution of concentration of interstitials; $D_{\rho}(x, T)$ are the diffusion coefficients of interstitials and vacancies; $k_{I, V}(x, T)$ is the parameter of recombination of point radiation defects; terms $V^{2}(x, y, z, t)$ and $I^{2}(x, y, z, t)$ correspond to generation of divacancies and analogous complexes of interstitials (see, for e.g., (Pankratov 2011) and appropriate references in this paper); $k_{I, V}(x, T)$ and $k_{\rho, \rho}(x, T)$ are the parameters of recombination of point defects and generation of their complexes, respectively; $k$ is the Boltzmann constant; $V^{*}$ is the equilibrium distribution of vacancies, $\omega=a^{3}, a$ is the atomic spacing; $\ell$ is the specific surface energy. To take into account porosity we assume that pores are approximately cylindrical with average dimensions $r=$ $\sqrt{x_{1}^{2}+y_{1}^{2}}$ and $z_{1}$ (Mynbaeva et al. 2008). The average size of the pores has been taken into account in boundary conditions on the pores in Eq. (5). With time, small pores decompose into vacancies. The vacancies are absorbed by large pores (Cheremskoy et al. 1990). With time, the large pores take spherical form during the absorbtion of vacancies from small pores (Cheremskoy et al. 1990). It was assumed that the pores are distributed initially homogenous with the appropriate concentration of vacancies, described the next relation (i.e., we determined distribution of concentration of vacancies, which was formed due to porosity, by summing overall pores)

$V(x, y, z, t)=\sum_{i=0}^{l} \sum_{j=0}^{m} \sum_{k=0}^{n} V_{p}(x+i \alpha, y+j \beta, z+k \chi, t)$.

Here $R=\sqrt{x^{2}+y^{2}+z^{2}} ; \alpha, \beta$ and $\chi$ are averaged distances between centers of pores in $x, y$ and $z$ directions, respectively; $l, m$ and $n$ are quantities of pores in the same directions; $i, j, k$ are current numbers of pores.

Spatiotemporal distribution of concentrations of divacancies $\Phi_{V}(x, y, z, t)$ and analogous complexes of interstitials $\Phi_{I}(x, y, z, t)$ could be determine by the following system of equations (Kitayama et al. 2000a, b; Vinetsky and Kholodar' 1979; Pankratov 2008b)

$$
\begin{aligned}
\frac{\partial \Phi_{\rho}(x, y, z, t)}{\partial t}= & \operatorname{div}\left\{D_{\Phi \rho}(x, T) \cdot \operatorname{grad}\left[\Phi_{\rho}(x, y, z, t)\right]\right\} \\
& +\operatorname{div}\left\{\frac{D_{\Phi_{\rho}}(x, T)}{\bar{V} k T} \cdot \operatorname{grad}[\mu(x, y, z, t)]\right\} \\
& +k_{\rho, \rho}(x, T) \rho^{2}(x, y, z, t) \\
& -k_{I}(x, T) \rho(x, y, z, t)
\end{aligned}
$$

with boundary and initial conditions

$$
\begin{aligned}
& \left.\frac{\partial \Phi_{\rho}(x, y, z, t)}{\partial x}\right|_{x=0}=0,\left.\quad \frac{\partial \Phi_{\rho}(x, y, z, t)}{\partial x}\right|_{x=L_{x}}=0, \\
& \left.\frac{\partial \Phi_{\rho}(x, y, z, t)}{\partial y}\right|_{y=0}=0,\left.\quad \frac{\partial \Phi_{\rho}(x, y, z, t)}{\partial y}\right|_{y=L_{y}}=0, \\
& \left.\frac{\partial \Phi_{\rho}(x, y, z, t)}{\partial z}\right|_{z=0}=0,\left.\quad \frac{\partial \Phi_{\rho}(x, y, z, t)}{\partial z}\right|_{z=L_{z}}=0, \\
& \Phi_{I}(x, y, z, 0)=f_{\Phi I}(x, y, z), \Phi_{V}(x, y, z, 0)=f_{\Phi V}(x, y, z) .
\end{aligned}
$$

Here $D_{\Phi I}(x, T)$ and $D_{\Phi V}(x, T)$ are the diffusion coefficients of complexes of point defects; $k_{I}(x, T)$ and $k_{V}(x, T)$ are the parameters of decay of complexes of point defects.

To determine spatiotemporal distributions of point defects in pursuance of Refs. Pankratov $(2005,2007)$, and Pankratov and Spagnolo (2005) we transform the approximations of diffusion coefficients of the defects in the following form: $D_{\rho}(x, T)=D_{0 \rho}\left[1+\varepsilon_{\rho} g_{\rho}(x, T)\right]$. In the same form we transform approximations of parameters of recombination of point defects and generation of their complexes: $k_{I, V}(x, T)=k_{0 I, V}\left[1+\varepsilon_{I, V} g_{I, V}(x, T)\right], k_{\rho, \rho}(x$, $T)=k_{0 \rho, \rho}\left[1+\varepsilon_{\rho, \rho} g_{\rho, \rho}(x, T)\right]$. Let us introduce the following dimensionless variables:

$$
\begin{aligned}
& \vartheta=\frac{t \sqrt{D_{0 I} D_{0 V}}}{L_{x}^{2}+L_{y}^{2}+L_{z}^{2}}, \chi=\frac{x}{L_{x}}, \eta=\frac{y}{L_{y}}, \phi=\frac{z}{L_{z}}, \\
& \omega=\left(L_{x}^{2}+L_{y}^{2}+L_{z}^{2}\right) \frac{I^{*} V^{*} k_{0 I, V}}{\sqrt{D_{0 I} D_{0 V}}}, b_{x}=1+\frac{L_{y}^{2}}{L_{x}^{2}}+\frac{L_{z}^{2}}{L_{x}^{2}}, \\
& b_{y}=1+\frac{L_{x}^{2}}{L_{y}^{2}}+\frac{L_{z}^{2}}{L_{y}^{2}}, b_{z}=1+\frac{L_{x}^{2}}{L_{z}^{2}}+\frac{L_{y}^{2}}{L_{z}^{2}}, \\
& \Omega_{\rho}=\left(\rho^{*}\right)^{2} k_{0 \rho, \rho} \frac{L_{x}^{2}+L_{y}^{2}+L_{z}^{2}}{\sqrt{D_{0 I} D_{0 V}}}, \tilde{I}(x, y, z, t)=\frac{I(x, y, z, t)}{I^{*}}, \\
& \tilde{V}(x, y, z, t)=\frac{V(x, y, z, t)}{V^{*}} .
\end{aligned}
$$

The change of variables gives us possibility to transform the Eq. (4) and condition Eq. (5) to the form

$$
\begin{aligned}
& \frac{\partial \tilde{I}(\chi, \eta, \phi, \vartheta)}{\partial \vartheta}=b_{x} \sqrt{\frac{D_{0 I}}{D_{0 V}}} \frac{\partial}{\partial \chi}\left\{\left[1+\varepsilon_{I} g_{I}(\chi, T)\right] \frac{\partial \tilde{I}(\chi, \eta, \phi, \vartheta)}{\partial \chi}\right\} \\
& +b_{y} \sqrt{\frac{D_{0 I}}{D_{0 V}}} \frac{\partial}{\partial \eta}\left\{\left[1+\varepsilon_{I} g_{I}(\chi, T)\right] \times \frac{\partial \tilde{I}(\chi, \eta, \phi, \vartheta)}{\partial \eta}\right\} \\
& +\frac{b_{x}}{\sqrt{D_{0 I} D_{0 V}}} \frac{\partial}{\partial \chi}\left[\frac{D_{I S}(\chi, T)}{\bar{V} k T} \frac{\partial \mu(\chi, \eta, \phi, \vartheta)}{\partial \chi}\right] \\
& +b_{z} \sqrt{\frac{D_{0 I}}{D_{0 V}}} \frac{\partial}{\partial \phi}\left\{\left[1+\varepsilon_{I} g_{I}(\chi, T)\right] \times \frac{\partial \tilde{I}(\chi, \eta, \phi, \vartheta)}{\partial \phi}\right\} \\
& +\frac{b_{y}}{\sqrt{D_{0 I} D_{0 V}}} \frac{\partial}{\partial \eta}\left[\frac{D_{I S}(\chi, T)}{\bar{V} k T} \frac{\partial \mu(\chi, \eta, \phi, \vartheta)}{\partial \eta}\right] \\
& +\frac{\partial}{\partial \phi}\left[\frac{D_{I S}(\chi, T)}{\bar{V} k T} \frac{\partial \mu(\chi, \eta, \phi, \vartheta)}{\partial \phi}\right] \\
& \quad \times \frac{b_{z}}{\sqrt{D_{0 I} D_{0 V}}}-\Omega_{I}\left[1+\varepsilon_{I, I} g_{I, I}(\chi, T)\right] \tilde{I}^{2}(\chi, \eta, \phi, \vartheta) \\
& -\omega \tilde{I}(\chi, \eta, \phi, \vartheta) \tilde{V}(\chi, \eta, \phi, \vartheta)\left[1+\varepsilon_{I, V} g_{I, V}(\chi, T)\right]
\end{aligned}
$$




$$
\begin{aligned}
& \frac{\partial \tilde{V}(\chi, \eta, \phi, \vartheta)}{\partial \vartheta}=b_{x} \sqrt{\frac{D_{0 V}}{D_{0 I}}} \frac{\partial}{\partial \chi}\left\{\left[1+\varepsilon_{V} g_{V}(\chi, T)\right] \frac{\partial \tilde{V}(\chi, \eta, \phi, \vartheta)}{\partial \chi}\right\} \\
& +b_{y} \sqrt{\frac{D_{0 V}}{D_{0 I}}} \frac{\partial}{\partial \eta}\left\{\frac{\partial \tilde{V}(\chi, \eta, \phi, \vartheta)}{\partial \eta} \times\left[1+\varepsilon_{V} g_{V}(\chi, T)\right]\right\} \\
& +b_{z} \sqrt{\frac{D_{0 V}}{D_{0 I}}} \frac{\partial}{\partial \phi}\left\{\left[1+\varepsilon_{V} g_{V}(\chi, T)\right] \frac{\partial \tilde{V}(\chi, \eta, \phi, \vartheta)}{\partial \phi}\right\} \\
& +\frac{\partial}{\partial \chi}\left[\frac{\partial \mu(\chi, \eta, \phi, \vartheta)}{\partial \chi} \times \frac{D_{V S}(\chi, T)}{\bar{V} k T}\right] \frac{b_{x}}{\sqrt{D_{0 I} D_{0 V}}} \\
& +\frac{b_{y}}{\sqrt{D_{0 I} D_{0 V}}} \frac{\partial}{\partial \eta}\left[\frac{D_{V S}(\chi, T)}{\bar{V} k T} \frac{\partial \mu(\chi, \eta, \phi, \vartheta)}{\partial \eta}\right] \\
& +\frac{b_{z}}{\sqrt{D_{0 I} D_{0 V}}} \frac{\partial}{\partial \phi}\left[\frac{\partial \mu(\chi, \eta, \phi, \vartheta)}{\partial \phi} \times \frac{D_{V S}(\chi, T)}{\bar{V} k T}\right] \\
& -\Omega_{V}\left[1+\varepsilon_{V, V} g_{V, V}(\chi, T)\right] \tilde{V}^{2}(\chi, \eta, \phi, \vartheta) \\
& -\omega \tilde{I}(\chi, \eta, \phi, \vartheta) \tilde{V}(\chi, \eta, \phi, \vartheta)\left[1+\varepsilon_{I, V} g_{I, V}(\chi, T)\right] \\
& \left.\frac{\partial \tilde{\rho}(\chi, \eta, \phi, \vartheta)}{\partial \chi}\right|_{\chi=0}=\left.\frac{\partial \tilde{\rho}(\chi, \eta, \phi, \vartheta)}{\partial \chi}\right|_{\chi=1}=0, \\
& \left.\frac{\partial \tilde{\rho}(\chi, \eta, \phi, \vartheta)}{\partial \eta}\right|_{\eta=0}=\left.\frac{\partial \tilde{\rho}(\chi, \eta, \phi, \vartheta)}{\partial \eta}\right|_{\eta=1}=0, \\
& \left.\frac{\partial \tilde{\rho}(\chi, \eta, \phi, \vartheta)}{\partial \phi}\right|_{\phi=0}=\left.\frac{\partial \tilde{\rho}(\chi, \eta, \phi, \vartheta)}{\partial \phi}\right|_{\phi=1}=0 \\
& \tilde{I}(\chi, \eta, \phi, \vartheta)=\frac{f_{I}(\chi, \eta, \phi, \vartheta)}{I^{*}} ; \tilde{V}(\chi, \eta, \phi, \vartheta)=\frac{f_{V}(\chi, \eta, \phi, \vartheta)}{V^{*}} .
\end{aligned}
$$

Let us determine solutions of Eq. (8) with condition Eq. (9), in pursuance of Refs. Pankratov (2005, 2007), and Pankratov and Spagnolo 2005), as the following power series

$\tilde{\rho}(\chi, \eta, \phi, \vartheta)=\sum_{i=0}^{\infty} \varepsilon_{\rho}^{i} \sum_{j=0}^{\infty} \omega^{j} \sum_{k=0}^{\infty} \Omega_{\rho}^{k} \tilde{\rho}_{i j k}(\chi, \eta, \phi, \vartheta)$.

Substitution of the series Eq. (10) into Eq. (8) and conditions Eq. (9) gives us possibility to obtain equations for zeroth-order approximations of point-defect concentrations $\tilde{I}_{000}(\chi, \eta, \phi, \vartheta)$ and $\tilde{V}_{000}(\chi, \eta, \phi, \vartheta)$ and corrections to the functions $\tilde{I}_{i j k}(\chi, \eta, \phi, \vartheta)$ and $\tilde{V}_{i j k}(\chi, \eta, \phi, \vartheta) \quad i \geq 1, j \geq 1$, $k \geq 1$. The equations and conditions are presented in the "Appendix". Equations of the system (11) could be solved by standard approaches of the mathematical physics (see, for e.g., Tikhonov and Samarskii 1972). The solutions are presented in the "Appendix".

Further we determine spatiotemporal distributions of complexes of radiation defects. To determine the distributions we transform the diffusion coefficients in the following form: $D_{\Phi I}(x, y, z, T)=D_{0 Ф I}\left[1+\varepsilon_{\Phi I} g_{\Phi I}(x, y, z, T)\right]$ and $D_{\Phi V}(x, y, z, T)=D_{0 \Phi V}\left[1+\varepsilon_{\Phi V} g_{\Phi V}(x, y, z, T)\right]$. In this situation the Eq. (6) takes the form

$$
\begin{aligned}
& \frac{\partial \Phi_{\rho}(x, y, z, t)}{\partial t}=\left(\frac{\partial}{\partial x}\left\{\left[1+\varepsilon_{\Phi \rho} g_{\Phi \rho}(x, T)\right] \frac{\partial \Phi_{\rho}(x, y, z, t)}{\partial x}\right\}\right. \\
& +\frac{\partial}{\partial y}\left\{\left[1+\varepsilon_{\Phi \rho} g_{\Phi \rho}(x, T)\right] \frac{\partial \Phi_{\rho}(x, y, z, t)}{\partial y}\right\} \\
& \left.+\frac{\partial}{\partial z}\left\{\left[1+\varepsilon_{\Phi \rho} g_{\Phi \rho}(x, T)\right] \frac{\partial \Phi_{\rho}(x, y, z, t)}{\partial z}\right\}\right) D_{0 \Phi \rho} \\
& +\frac{\partial}{\partial x}\left[\frac{D_{\Phi_{\rho} S}(x, T)}{\bar{V} k T} \frac{\partial \mu(x, y, z, t)}{\partial x}\right]-\rho(x, y, z, t) \\
& \quad \times k_{\rho}(x, T)+\frac{\partial}{\partial y}\left[\frac{D_{\Phi_{\rho}}(x, T)}{\bar{V} k T} \frac{\partial \mu(x, y, z, t)}{\partial y}\right] \\
& +\frac{\partial}{\partial z}\left[\frac{D_{\Phi_{\rho} S}(x, T)}{\bar{V} k T} \frac{\partial \mu(x, y, z, t)}{\partial z}\right]+k_{\rho, \rho}(x, T) \rho^{2}(x, y, z, t) .
\end{aligned}
$$

Let us determine solution of the equations as the power series

$\Phi_{\rho}(x, y, z, t)=\sum_{i=0}^{\infty} \varepsilon_{\Phi \rho}^{i} \Phi_{\rho i}(x, y, z, t)$.

Substitution of the series Eq. (11) into Eq. (6) and appropriate boundary and initial conditions give us possibility to zero-order approximations of concentrations of complexes of radiation defects, corrections and conditions for them. The equations, conditions for them and their solutions are presented in the "Appendix".

Let us determine spatiotemporal distribution of dopant concentration using the same approach as for determination of spatiotemporal distribution of radiation defects concentration. We transform approximation of dopant diffusion coefficient to the following form: $D_{L}(x, T)=$ $D_{0 L}\left[1+\varepsilon_{L} g_{L}(x, T)\right]$. Further we determine solution of the Eq. (1) as the following power series

$C(x, y, z, t)=\sum_{i=0}^{\infty} \varepsilon_{L}^{i} \sum_{\xi=1}^{\infty} \xi^{j} C_{i j}(x, y, z, t)$.

Substitution of the series in the Eqs. (1) and (2) gives us possibility to obtain equations for zero-order approximation of the dopant concentration $C_{00}(x, y, z, t)$, corrections to it $C_{i j}(x, y, z, t)$ and boundary and initial conditions to them. The equations, conditions for them and their solutions are presented in the "Appendix".

Analysis of spatiotemporal distributions of dopant and radiation defect concentrations has been done analytically using the second-order approximation of the dopant concentration. Further the distribution has been amended numerically.

\section{Discussion}

In the previous section we obtain relation to describe spatiotemporal distributions of radiation defects and dopant concentrations. It has been recently shown, that implantation of ions of dopant in a $\mathrm{H}$ gives us possibility to increase 
sharpness of $\mathrm{p}-\mathrm{n}$-junction and to increase homogeneity of dopant distribution in doped area (Pankratov 2008a, 2010a, b, 2011). To obtain the both effects at one time it is necessary to optimize annealing time $\theta$. Framework the optimization we approximate real spatial distributions of dopant and minimizing the following mean-squared error

$U=\frac{1}{L} \int_{0}^{L}[C(x, t)-\psi(x)]^{2} \mathrm{~d} x$,

where $C(x, t)$ is the real spatiotemporal distribution of dopant concentration, $\psi(x)$ is the step-wise approximation of the concentration. The optimization procedure has been described in several previous works and (at the same time the optimization is not the main result of the work) will not be presented in the paper. The result of optimization is illustrated by Fig. 2 . In this figure it has been compared spatial distributions of dopant in homogenous sample and in $\mathrm{H}$ with experimental one.

The main aim of the present paper is analysis of the possibility to decrease quantity of radiation defects. To decrease the quantity of radiation defects some approaches (such as different types of annealing (Lachin and Savelov 2001; Gotra 1991; Bykov et al. 2003; Ong et al. 2006; Ahlgren et al. 1997; Noda 2003), annealing in combination with multistage ion implantation (Pankratov and Bulaeva 2011)) could be used. In this paper we consider a $\mathrm{H}$, which consist of substrate and porous epitaxial layer. In this paper we analyzed spatiotemporal distributions of concentrations of radiation defects and dopant using modified method of small parameter (we used main idea of method of small parameter, but parameters, which used in appropriate power series could correspond to enough large variations of diffusion coefficients, and

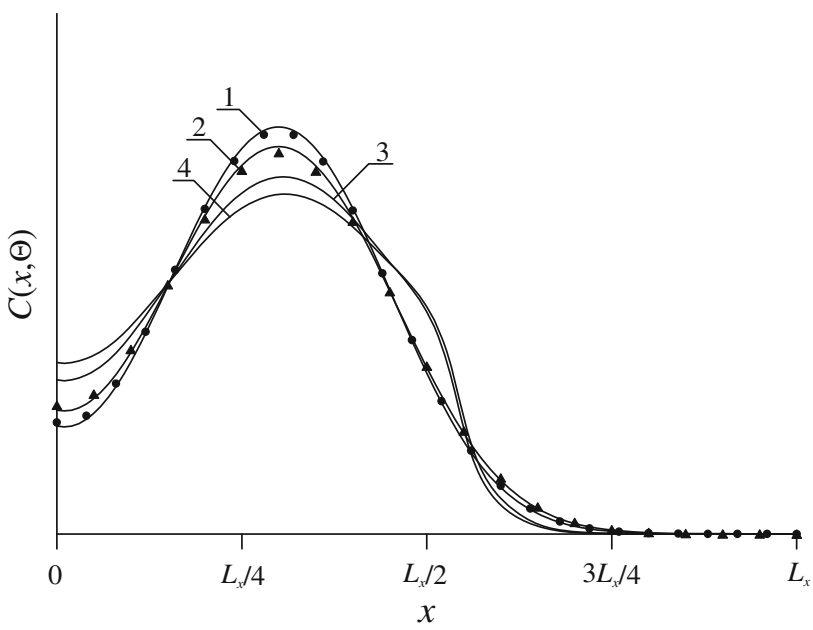

Fig. 2 Distributions of dopant concentration after annealing of radiation defects with continuance $\Theta=0.0048\left(L_{x}^{2}+L_{y}^{2}+L_{z}^{2}\right) / D_{0 L}$ (curves 1 and 3) and $\Theta=0.0057\left(L_{x}^{2}+L_{y}^{2}+L_{z}^{2}\right) / D_{0 L}$ (curves 2 and 4), where $D_{0 L}$ is average value of diffusion coefficient. Circles and triangles are experimental data from (Ahlgren et al. 1997; Noda 2003). Interface between layers of $H$ has coordinate $a_{x}=L_{x} / 2$

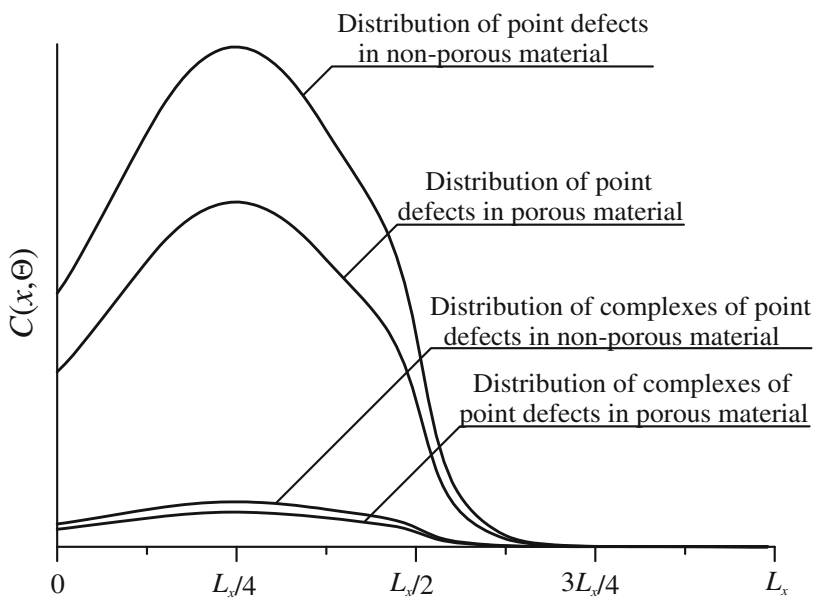

Fig. 3 Distributions of concentrations of point radiation defects and their complexes after annealing with time $\Theta=0.005\left(L_{x}^{2}+L_{y}^{2}+L_{z}^{2}\right) /$ $D_{0 L}$. Curves for complexes of defects have been increased into 50 times. Interface between layers of $\mathrm{H}$ has coordinate $a_{x}=L_{x} / 2$

parameters of recombination of defects, generation of complexes of defects and decay of complexes of defects) (Pankratov 2005, 2007; Pankratov and Spagnolo 2005; Pankratov and Bulaeva 2011). We obtained, that porosity of EL gives us possibility to decrease quantity of radiation defects. In Fig. 3 we compare distributions of concentrations of radiation defects in porous EL and in non-porous EL. Probably, radiation defects leave to the pores from their neighborhoods.

In our model we take into account diffusion of dopant and radiation defects, recombination of point radiation defects, generation and decay of their simplest complexes (interstitials and divacancies). We also take into account non-linearity of dopant diffusion for high-doped materials.

\section{Conclusion}

In this paper we consider an approach to increase sharpness of the implanted-junction rectifier in a semiconductor heterostructure, which consist of two layers (substrate and epitaxial layer). At the same time with increase of the sharpness homogeneity of dopant distribution in doped area increases. We obtain that maximal compromise between the effects could be obtained, when $\mathrm{p}-\mathrm{n}$-junction has been fabricated near interface between layers of the heterostructure. In this paper we introduce an approach to decrease quantity of radiation defects using porosity of epitaxial layer. In this case the radiation defects, probably, leave to the pores from their neighborhoods.

Acknowledgments This work has been supported by grant of President of Russia (project MK-548.2010.2).

Open Access This article is distributed under the terms of the Creative Commons Attribution License which permits any use, distribution, and reproduction in any medium, provided the original author(s) and the source are credited. 


\section{Appendix}

Equations for the functions $\tilde{I}_{i j k}(\chi, \eta, \phi, \vartheta), \tilde{V}_{i j k}(\chi, \eta, \phi, \vartheta)$, ( $i \geq 0, j \geq 0, k \geq 0$ ), and boundary and initial conditions for them could be written as

$$
\begin{aligned}
& \frac{\partial \tilde{I}_{000}(\chi, \eta, \phi, \vartheta)}{\partial \vartheta}= \sqrt{\frac{D_{0 I}}{D_{0 V}}}\left[b_{x} \frac{\partial^{2} \tilde{I}_{000}(\chi, \eta, \phi, \vartheta)}{\partial \chi^{2}}+b_{y} \frac{\partial^{2} \tilde{I}_{000}(\chi, \eta, \phi, \vartheta)}{\partial \eta^{2}}+b_{z} \frac{\partial^{2} \tilde{I}_{000}(\chi, \eta, \phi, \vartheta)}{\partial \phi^{2}}\right]+\frac{1}{\sqrt{D_{0 I} D_{0 V}}} \\
& \times\left\{b_{x} \frac{\partial}{\partial \chi}\left[\frac{D_{\mathrm{IS}}(\chi, T)}{\bar{V} k T} \frac{\partial \mu(\chi, \eta, \phi, \vartheta)}{\partial \chi}\right]+b_{y} \frac{\partial}{\partial \eta}\left[\frac{D_{\mathrm{IS}}(\chi, T)}{\bar{V} k T} \frac{\partial \mu(\chi, \eta, \phi, \vartheta)}{\partial \eta}\right]+b_{z} \frac{\partial}{\partial \phi}\left[\frac{D_{\mathrm{IS}}(\chi, T)}{\bar{V} k T} \frac{\partial(\chi, \eta, \phi, \vartheta)}{\partial \phi}\right]\right\} ; \\
& \frac{\partial \tilde{V}_{000}(\chi, \eta, \phi, \vartheta)}{\partial \vartheta}= \sqrt{\frac{D_{0 V}}{D_{0 I}}}\left[b_{x} \frac{\partial^{2} \tilde{V}_{000}(\chi, \eta, \phi, \vartheta)}{\partial \chi^{2}}+b_{y} \frac{\partial^{2} \tilde{V}_{000}(\chi, \eta, \phi, \vartheta)}{\partial \eta^{2}}+b_{z} \frac{\partial^{2} \tilde{V}_{000}(\chi, \eta, \phi, \vartheta)}{\partial \phi^{2}}\right]+\frac{1}{\sqrt{D_{0 I} D_{0 V}}} \\
&+\frac{1}{\sqrt{D_{0 I} D_{0 V}}}\left\{b_{x} \frac{\partial}{\partial \chi}\left[\frac{D_{\mathrm{VS}}(\chi, T)}{\bar{V} k T} \frac{\partial \mu(\chi, \eta, \phi, \vartheta)}{\partial \chi}\right]+b_{y} \frac{\partial}{\partial \eta}\left[\frac{D_{\mathrm{VS}}(\chi, T)}{\bar{V} k T} \frac{\partial(\chi, \eta, \phi, \vartheta)}{\partial \eta}\right]\right. \\
&\left.+b_{z} \frac{\partial}{\partial \phi}\left[\frac{D_{\mathrm{VS}}(\chi, T)}{\bar{V} k T} \frac{\partial \mu(\chi, \eta, \phi, \vartheta)}{\partial \phi}\right]\right\} ;
\end{aligned}
$$

$$
\begin{aligned}
\frac{\partial \tilde{I}_{i 00}(\chi, \eta, \phi, \vartheta)}{\partial \vartheta}= & \sqrt{\frac{D_{0 I}}{D_{0 V}}}\left[b_{x} \frac{\partial^{2} \tilde{I}_{i 00}(\chi, \eta, \phi, \vartheta)}{\partial \chi^{2}}+b_{y} \frac{\partial^{2} \tilde{I}_{i 00}(\chi, \eta, \phi, \vartheta)}{\partial \eta^{2}}+b_{z} \frac{\partial^{2} \tilde{I}_{i 00}(\chi, \eta, \phi, \vartheta)}{\partial \phi^{2}}\right] \\
& +\sqrt{\frac{D_{0 I}}{D_{0 V}}}\left\{b_{x} \frac{\partial}{\partial \chi}\left[g_{I}(\chi, T) \frac{\partial \tilde{I}_{i-100}(\chi, \eta, \phi, \vartheta)}{\partial \chi}\right]+b_{y} \frac{\partial}{\partial \eta}\left[g_{I}(\chi, T) \frac{\partial \tilde{I}_{i-100}(\chi, \eta, \phi, \vartheta)}{\partial \eta}\right]\right. \\
& \left.+b_{z} \frac{\partial}{\partial \phi}\left[g_{I}(\chi, T) \frac{\partial \tilde{I}_{i-100}(\chi, \eta, \phi, \vartheta)}{\partial \phi}\right]\right\}, i \geq 1
\end{aligned}
$$

$$
\begin{aligned}
\frac{\partial \tilde{V}_{i 00}(\chi, \eta, \phi, \vartheta)}{\partial \vartheta}= & \sqrt{\frac{D_{0 V}}{D_{0 I}}}\left[b_{x} \frac{\partial^{2} \tilde{V}_{i 00}(\chi, \eta, \phi, \vartheta)}{\partial \chi^{2}}+b_{y} \frac{\partial^{2} \tilde{V}_{i 00}(\chi, \eta, \phi, \vartheta)}{\partial \eta^{2}}+b_{z} \frac{\partial^{2} \tilde{V}_{i 00}(\chi, \eta, \phi, \vartheta)}{\partial \phi^{2}}\right] \\
& +\sqrt{\frac{D_{0 V}}{D_{0 I}}}\left\{b_{x} \frac{\partial}{\partial \chi}\left[g_{V}(\chi, T) \frac{\partial \tilde{V}_{i-100}(\chi, \eta, \phi, \vartheta)}{\partial \chi}\right]+b_{y} \frac{\partial}{\partial \eta}\left[g_{V}(\chi, T) \frac{\partial \tilde{V}_{i-100}(\chi, \eta, \phi, \vartheta)}{\partial \eta}\right]\right. \\
& \left.+b_{z} \frac{\partial}{\partial \phi}\left[g_{V}(\chi, T) \frac{\partial \tilde{V}_{i-100}(\chi, \eta, \phi, \vartheta)}{\partial \phi}\right]\right\}, i \geq 1 ;
\end{aligned}
$$

$\frac{\partial \tilde{I}_{010}(\chi, \eta, \phi, \vartheta)}{\partial \vartheta}=\sqrt{\frac{D_{0 I}}{D_{0 V}}}\left[b_{x} \frac{\partial^{2} \tilde{I}_{010}(\chi, \eta, \phi, \vartheta)}{\partial \chi^{2}}+b_{y} \frac{\partial^{2} \tilde{I}_{010}(\chi, \eta, \phi, \vartheta)}{\partial \eta^{2}}+b_{z} \frac{\partial^{2} \tilde{I}_{010}(\chi, \eta, \phi, \vartheta)}{\partial \phi^{2}}\right]$

$$
-\left[1+\varepsilon_{I, V} g_{I, V}(\chi, T)\right] \tilde{I}_{000}(\chi, \eta, \phi, \vartheta) \tilde{V}_{000}(\chi, \eta, \phi, \vartheta) ;
$$




$$
\begin{aligned}
\frac{\partial \tilde{V}_{010}(\chi, \eta, \phi, \vartheta)}{\partial \vartheta}= & \sqrt{\frac{D_{0 V}}{D_{0 I}}}\left[b_{x} \frac{\partial^{2} \tilde{V}_{010}(\chi, \eta, \phi, \vartheta)}{\partial \chi^{2}}+b_{y} \frac{\partial^{2} \tilde{V}_{010}(\chi, \eta, \phi, \vartheta)}{\partial \eta^{2}}+b_{z} \frac{\partial^{2} \tilde{V}_{010}(\chi, \eta, \phi, \vartheta)}{\partial \phi^{2}}\right] \\
& -\left[1+\varepsilon_{I, V} g_{I, V}(\chi, T)\right] \tilde{I}_{000}(\chi, \eta, \phi, \vartheta) \tilde{V}_{000}(\chi, \eta, \phi, \vartheta)
\end{aligned}
$$$$
\frac{\partial \tilde{I}_{020}(\chi, \eta, \phi, \vartheta)}{\partial \vartheta}=\sqrt{\frac{D_{0 I}}{D_{0 V}}}\left[b_{x} \frac{\partial^{2} \tilde{I}_{020}(\chi, \eta, \phi, \vartheta)}{\partial \chi^{2}}+b_{y} \frac{\partial^{2} \tilde{I}_{020}(\chi, \eta, \phi, \vartheta)}{\partial \eta^{2}}+b_{z} \frac{\partial^{2} \tilde{I}_{020}(\chi, \eta, \phi, \vartheta)}{\partial \phi^{2}}\right]
$$$$
-\left[1+\varepsilon_{I, V} g_{I, V}(\chi, T)\right]\left[\tilde{I}_{010}(\chi, \eta, \phi, \vartheta) \tilde{V}_{000}(\chi, \eta, \phi, \vartheta)+\tilde{I}_{000}(\chi, \eta, \phi, \vartheta) \tilde{V}_{010}(\chi, \eta, \phi, \vartheta)\right] \text {; }
$$

$$
\begin{aligned}
\frac{\partial \tilde{V}_{020}(\chi, \eta, \phi, \vartheta)}{\partial \vartheta}= & \sqrt{\frac{D_{0 V}}{D_{0 I}}}\left[b_{x} \frac{\partial^{2} \tilde{V}_{020}(\chi, \eta, \phi, \vartheta)}{\partial \chi^{2}}+b_{y} \frac{\partial^{2} \tilde{V}_{020}(\chi, \eta, \phi, \vartheta)}{\partial \eta^{2}}+b_{z} \frac{\partial^{2} \tilde{V}_{020}(\chi, \eta, \phi, \vartheta)}{\partial \phi^{2}}\right] \\
& -\left[\tilde{I}_{010}(\chi, \eta, \phi, \vartheta) \tilde{V}_{000}(\chi, \eta, \phi, \vartheta)+\tilde{I}_{000}(\chi, \eta, \phi, \vartheta) \tilde{V}_{010}(\chi, \eta, \phi, \vartheta)\right]\left[1+\varepsilon_{I, V} g_{I, V}(\chi, \eta, \phi, T)\right] ;
\end{aligned}
$$

$$
\begin{aligned}
\frac{\partial \tilde{I}_{001}(\chi, \eta, \phi, \vartheta)}{\partial \vartheta}= & \sqrt{\frac{D_{0 I}}{D_{0 V}}}\left[b_{x} \frac{\partial^{2} \tilde{I}_{001}(\chi, \eta, \phi, \vartheta)}{\partial \chi^{2}}+b_{y} \frac{\partial^{2} \tilde{I}_{001}(\chi, \eta, \phi, \vartheta)}{\partial \eta^{2}}+b_{z} \frac{\partial^{2} \tilde{I}_{001}(\chi, \eta, \phi, \vartheta)}{\partial \phi^{2}}\right] \\
& -\left[1+\varepsilon_{I, I} g_{I, I}(\chi, T)\right] \tilde{I}_{000}^{2}(\chi, \eta, \phi, \vartheta) ;
\end{aligned}
$$

$$
\begin{aligned}
\frac{\partial \tilde{V}_{001}(\chi, \eta, \phi, \vartheta)}{\partial \vartheta}= & \sqrt{\frac{D_{0 V}}{D_{0 I}}}\left[b_{x} \frac{\partial^{2} \tilde{V}_{001}(\chi, \eta, \phi, \vartheta)}{\partial \chi^{2}}+b_{y} \frac{\partial^{2} \tilde{V}_{001}(\chi, \eta, \phi, \vartheta)}{\partial \eta^{2}}+b_{z} \frac{\partial^{2} \tilde{V}_{001}(\chi, \eta, \phi, \vartheta)}{\partial \phi^{2}}\right] \\
& -\left[1+\varepsilon_{V, V} g_{V, V}(\chi, T)\right] \tilde{V}_{000}^{2}(\chi, \eta, \phi, \vartheta) ;
\end{aligned}
$$$$
\frac{\partial \tilde{I}_{002}(\chi, \eta, \phi, \vartheta)}{\partial \vartheta}=\sqrt{\frac{D_{0 I}}{D_{0 V}}}\left[b_{x} \frac{\partial^{2} \tilde{I}_{002}(\chi, \eta, \phi, \vartheta)}{\partial \chi^{2}}+b_{y} \frac{\partial^{2} \tilde{I}_{002}(\chi, \eta, \phi, \vartheta)}{\partial \eta^{2}}+b_{z} \frac{\partial^{2} \tilde{I}_{002}(\chi, \eta, \phi, \vartheta)}{\partial \phi^{2}}\right]
$$$$
-\left[1+\varepsilon_{I, I} g_{I, I}(\chi, T)\right] \tilde{I}_{001}(\chi, \eta, \phi, \vartheta) \tilde{I}_{000}(\chi, \eta, \phi, \vartheta) ;
$$

$$
\begin{aligned}
\frac{\partial \tilde{V}_{002}(\chi, \eta, \phi, \vartheta)}{\partial \vartheta}= & \sqrt{\frac{D_{0 V}}{D_{0 I}}}\left[b_{x} \frac{\partial^{2} \tilde{V}_{002}(\chi, \eta, \phi, \vartheta)}{\partial \chi^{2}}+b_{y} \frac{\partial^{2} \tilde{V}_{002}(\chi, \eta, \phi, \vartheta)}{\partial \eta^{2}}+b_{z} \frac{\partial^{2} \tilde{V}_{002}(\chi, \eta, \phi, \vartheta)}{\partial \phi^{2}}\right] \\
& -\left[1+\varepsilon_{V, V} g_{V, V}(\chi, T)\right] \tilde{V}_{001}(\chi, \eta, \phi, \vartheta) \tilde{V}_{000}(\chi, \eta, \phi, \vartheta) ;
\end{aligned}
$$

$$
\begin{aligned}
\frac{\partial \tilde{I}_{110}(\chi, \eta, \phi, \vartheta)}{\partial \vartheta}= & \sqrt{\frac{D_{0 I}}{D_{0 V}}}\left[b_{x} \frac{\partial^{2} \tilde{I}_{110}(\chi, \eta, \phi, \vartheta)}{\partial \chi^{2}}+b_{y} \frac{\partial^{2} \tilde{I}_{110}(\chi, \eta, \phi, \vartheta)}{\partial \eta^{2}}+b_{z} \frac{\partial^{2} \tilde{I}_{110}(\chi, \eta, \phi, \vartheta)}{\partial \phi^{2}}\right] \\
& +\sqrt{\frac{D_{0 I}}{D_{0 V}}}\left\{b_{x} \frac{\partial}{\partial \chi}\left[g_{I}(\chi, T) \frac{\partial \tilde{I}_{010}(\chi, \eta, \phi, \vartheta)}{\partial \chi}\right]+b_{y} \frac{\partial}{\partial \eta}\left[g_{I}(\chi, T) \frac{\partial \tilde{I}_{010}(\chi, \eta, \phi, \vartheta)}{\partial \eta}\right]\right. \\
& \left.+b_{z} \frac{\partial}{\partial \phi}\left[g_{I}(\chi, T) \frac{\partial \tilde{I}_{010}(\chi, \eta, \phi, \vartheta)}{\partial \phi}\right]\right\}-\left[1+\varepsilon_{I I I} g_{I, I}(\chi, T)\right]\left[\tilde{I}_{100}(\chi, \eta, \phi, \vartheta) \tilde{V}_{000}(\chi, \eta, \phi, \vartheta)\right. \\
& \left.+\tilde{I}_{000}(\chi, \eta, \phi, \vartheta) \tilde{V}_{100}(\chi, \eta, \phi, \vartheta)\right] ;
\end{aligned}
$$

$$
\begin{aligned}
\frac{\partial \tilde{V}_{110}(\chi, \eta, \phi, \vartheta)}{\partial \vartheta}= & \sqrt{\frac{D_{0 V}}{D_{0 I}}}\left[b_{x} \frac{\partial^{2} \tilde{V}_{110}(\chi, \eta, \phi, \vartheta)}{\partial \chi^{2}}+b_{y} \frac{\partial^{2} \tilde{V}_{110}(\chi, \eta, \phi, \vartheta)}{\partial \eta^{2}}+b_{z} \frac{\partial^{2} \tilde{V}_{110}(\chi, \eta, \phi, \vartheta)}{\partial \phi^{2}}\right]+\sqrt{\frac{D_{0 V}}{D_{0 I}}} \\
& \times\left\{b_{x} \frac{\partial}{\partial \chi}\left[g_{V}(\chi, T) \frac{\partial \tilde{V}_{010}(\chi, \eta, \phi, \vartheta)}{\partial \chi}\right]+b_{y} \frac{\partial}{\partial \eta}\left[g_{V}(\chi, T) \frac{\partial \tilde{V}_{010}(\chi, \eta, \phi, \vartheta)}{\partial \eta}\right]+b_{z} \frac{\partial}{\partial \phi}\left[\frac{\partial \tilde{V}_{010}(\chi, \eta, \phi, \vartheta)}{\partial \phi}\right.\right. \\
& \left.\left.\times g_{V}(\chi, T)\right]\right\}-\left[1+\varepsilon_{V, V} g_{V, V}(\chi, T)\right]\left[\tilde{I}_{100}(\chi, \eta, \phi, \vartheta) \tilde{V}_{000}(\chi, \eta, \phi, \vartheta)+\tilde{I}_{000}(\chi, \eta, \phi, \vartheta) \tilde{V}_{100}(\chi, \eta, \phi, \vartheta)\right] ;
\end{aligned}
$$




$$
\begin{aligned}
\frac{\partial \tilde{I}_{101}(\chi, \eta, \phi, \vartheta)}{\partial \vartheta}= & \sqrt{\frac{D_{0 I}}{D_{0 V}}}\left[b_{x} \frac{\partial^{2} \tilde{I}_{101}(\chi, \eta, \phi, \vartheta)}{\partial \chi^{2}}+b_{y} \frac{\partial^{2} \tilde{I}_{101}(\chi, \eta, \phi, \vartheta)}{\partial \eta^{2}}+b_{y} \frac{\partial^{2} \tilde{I}_{101}(\chi, \eta, \phi, \vartheta)}{\partial \phi^{2}}\right] \\
& +\sqrt{\frac{D_{0 I}}{D_{0 V}}}\left\{b_{x} \frac{\partial}{\partial \chi}\left[g_{I}(\chi, T) \frac{\partial \tilde{I}_{001}(\chi, \eta, \phi, \vartheta)}{\partial \chi}\right]+b_{y} \frac{\partial}{\partial \eta}\left[g_{I}(\chi, T) \frac{\partial \tilde{I}_{001}(\chi, \eta, \phi, \vartheta)}{\partial \eta}\right]\right. \\
& \left.+b_{z} \frac{\partial}{\partial \phi}\left[g_{I}(\chi, T) \frac{\partial \tilde{I}_{001}(\chi, \eta, \phi, \vartheta)}{\partial \phi}\right]\right\}-\left[1+\varepsilon_{I} g_{I}(\chi, T)\right] \tilde{I}_{100}(\chi, \eta, \phi, \vartheta) \tilde{V}_{000}(\chi, \eta, \phi, \vartheta)
\end{aligned}
$$

$\frac{\partial \tilde{V}_{101}(\chi, \eta, \phi, \vartheta)}{\partial \vartheta}=\sqrt{\frac{D_{0 V}}{D_{0 I}}}\left[b_{x} \frac{\partial^{2} \tilde{V}_{101}(\chi, \eta, \phi, \vartheta)}{\partial \chi^{2}}+b_{y} \frac{\partial^{2} \tilde{V}_{101}(\chi, \eta, \phi, \vartheta)}{\partial \eta^{2}}+b_{y} \frac{\partial^{2} \tilde{V}_{101}(\chi, \eta, \phi, \vartheta)}{\partial \phi^{2}}\right]$

$$
\begin{aligned}
& +\sqrt{\frac{D_{0 V}}{D_{0 I}}}\left\{b_{x} \frac{\partial}{\partial \chi}\left[g_{V}(\chi, T) \frac{\partial \tilde{V}_{001}(\chi, \eta, \phi, \vartheta)}{\partial \chi}\right]+b_{y} \frac{\partial}{\partial \eta}\left[g_{V}(\chi, T) \frac{\partial \tilde{V}_{001}(\chi, \eta, \phi, \vartheta)}{\partial \eta}\right]\right. \\
& \left.+b_{z} \frac{\partial}{\partial \phi}\left[g_{V}(\chi, T) \frac{\partial \tilde{V}_{001}(\chi, \eta, \phi, \vartheta)}{\partial \phi}\right]\right\}-\left[1+\varepsilon_{I} g_{I}(\chi, T)\right] \tilde{I}_{100}(\chi, \eta, \phi, \vartheta) \tilde{V}_{000}(\chi, \eta, \phi, \vartheta) ;
\end{aligned}
$$

$\frac{\partial \tilde{I}_{011}(\chi, \eta, \phi, \vartheta)}{\partial \vartheta}=\sqrt{\frac{D_{0 I}}{D_{0 V}}}\left[b_{x} \frac{\partial^{2} \tilde{I}_{011}(\chi, \eta, \phi, \vartheta)}{\partial \chi^{2}}+b_{y} \frac{\partial^{2} \tilde{I}_{011}(\chi, \eta, \phi, \vartheta)}{\partial \eta^{2}}+b_{z} \frac{\partial^{2} \tilde{I}_{011}(\chi, \eta, \phi, \vartheta)}{\partial \phi^{2}}\right]$

$-\left[1+\varepsilon_{I, I} g_{I, I}(\chi, T)\right] \tilde{I}_{000}(\chi, \eta, \phi, \vartheta) \tilde{I}_{010}(\chi, \eta, \phi, \vartheta)-\left[1+\varepsilon_{I, V} g_{I, V}(\chi, T)\right] \tilde{I}_{001}(\chi, \eta, \phi, \vartheta) \tilde{V}_{000}(\chi, \eta, \phi, \vartheta) ;$

$\frac{\partial \tilde{V}_{011}(\chi, \eta, \phi, \vartheta)}{\partial \vartheta}=\sqrt{\frac{D_{0 V}}{D_{0 I}}}\left[b_{x} \frac{\partial^{2} \tilde{V}_{011}(\chi, \eta, \phi, \vartheta)}{\partial \chi^{2}}+b_{y} \frac{\partial^{2} \tilde{V}_{011}(\chi, \eta, \phi, \vartheta)}{\partial \eta^{2}}+b_{z} \frac{\partial^{2} \tilde{V}_{011}(\chi, \eta, \phi, \vartheta)}{\partial \phi^{2}}\right]$

$-\left[1+\varepsilon_{I, I} g_{I, I}(\chi, T)\right] \tilde{I}_{000}(\chi, \eta, \phi, \vartheta) \tilde{I}_{010}(\chi, \eta, \phi, \vartheta)$

$-\left[1+\varepsilon_{I, V} g_{I, V}(\chi, T)\right] \tilde{I}_{001}(\chi, \eta, \phi, \vartheta) \tilde{V}_{000}(\chi, \eta, \phi, \vartheta) ;$

$\left.\frac{\partial \tilde{\rho}_{i j k}(\chi, \eta, \phi, \vartheta)}{\partial \chi}\right|_{x=0}=0,\left.\quad \frac{\partial \tilde{\rho}_{i j k}(\chi, \eta, \phi, \vartheta)}{\partial \chi}\right|_{x=1}=0,\left.\quad \frac{\partial \tilde{\rho}_{i j k}(\chi, \eta, \phi, \vartheta)}{\partial \eta}\right|_{\eta=0}=0,\left.\quad \frac{\partial \tilde{\rho}_{i j k}(\chi, \eta, \phi, \vartheta)}{\partial \eta}\right|_{\eta=1}=0$,

$\left.\frac{\partial \tilde{\rho}_{i j k}(\chi, \eta, \phi, \vartheta)}{\partial \phi}\right|_{\phi=0}=0,\left.\quad \frac{\partial \tilde{\rho}_{i j k}(\chi, \eta, \phi, \vartheta)}{\partial \phi}\right|_{\phi=1}=0,\left.\quad \frac{\partial \tilde{I}_{i j k}(\chi, \eta, \phi, \vartheta)}{\partial \chi}\right|_{x=\frac{x_{1}}{L_{x}}}=0,\left.\quad \frac{\partial \tilde{I}_{i j k}(\chi, \eta, \phi, \vartheta)}{\partial \eta}\right|_{\eta=\frac{y_{1}}{L_{y}}}=0$, $\tilde{V}_{000}\left(\frac{x_{1}}{L_{x}}+\vartheta \frac{V_{n}}{L_{x}} \frac{L_{x}^{2}+L_{y}^{2}+L_{z}^{2}}{\sqrt{D_{0 I} D_{0 V}}}, \frac{y_{1}}{L_{y}}+\vartheta \frac{V_{n}}{L_{y}} \frac{L_{x}^{2}+L_{y}^{2}+L_{z}^{2}}{\sqrt{D_{0 I} D_{0 V}}}, \frac{z_{1}}{L_{z}}+\vartheta \frac{V_{n}}{L_{z}} \frac{L_{x}^{2}+L_{y}^{2}+L_{z}^{2}}{\sqrt{D_{0 I} D_{0 V}}}, \vartheta\right)=1+\frac{2 \ell \omega}{k T \sqrt{x_{1}^{2}+y_{1}^{2}+z_{1}^{2}}}$, $\tilde{V}_{i j k}\left(\frac{x_{1}}{L_{x}}+\vartheta \frac{V_{n}}{L_{x}} \frac{L_{x}^{2}+L_{y}^{2}+L_{z}^{2}}{\sqrt{D_{0 I} D_{0 V}}}, \frac{y_{1}}{L_{y}}+\vartheta \frac{V_{n}}{L_{y}} \frac{L_{x}^{2}+L_{y}^{2}+L_{z}^{2}}{\sqrt{D_{0 I} D_{0 V}}}, \frac{z_{1}}{L_{z}}+\vartheta \frac{V_{n}}{L_{z}} \frac{L_{x}^{2}+L_{y}^{2}+L_{z}^{2}}{\sqrt{D_{0 I} D_{0 V}}}, \vartheta\right)=0, \quad(i \geq 1, j \geq 1, k \geq 1) ;$ $\left.\frac{\partial \tilde{I}_{i j k}(\chi, \eta, \phi, \vartheta)}{\partial \phi}\right|_{\phi=\frac{z_{1}}{L_{z}}}=0,(i \geq 1, j \geq 1, k \geq 1) ; \tilde{\rho}_{000}(\chi, \eta, \phi, 0)=\frac{f_{\rho}(\chi, \eta, \phi)}{\rho^{*}}, \tilde{\rho}_{i j k}(\chi, \eta, \phi, 0)=0$

$$
(i \geq 1, j \geq 1, k \geq 1)
$$

Solutions of the above equations with accounted boundary and initial conditions are 


$$
\begin{aligned}
& \tilde{I}_{000}(\chi, \eta, \phi, \vartheta)=-\frac{2 \pi b_{x}}{\sqrt{D_{0 I} D_{0 V}}} \sum_{n=1}^{\infty} n C_{n}(\chi, \eta, \phi) e_{n I}(\vartheta) \int_{0}^{\vartheta} e_{n I}(-\tau) \int_{0}^{1} \sin (\pi n u) \int_{0}^{1} \cos (\pi n v) \int_{0}^{1} \cos (\pi n w)\left[F_{n I}\right. \\
& \left.+\frac{D_{I S}(u, T)}{\bar{V} k T} \frac{\partial \mu(u, v, w, \tau)}{\partial u}\right] \mathrm{d} w \mathrm{~d} v \mathrm{~d} u \mathrm{~d} \tau-\sum_{n=1}^{\infty} n C_{n}(\chi, \eta, \phi) e_{n I}(\vartheta) \int_{0}^{\vartheta} e_{n I}(-\tau) \int_{0}^{1} \cos (\pi n u) \int_{0}^{1} \sin (\pi n v) \\
& \times \int_{0}^{1} \cos (\pi n w)\left[F_{n I}+\frac{D_{I S}(u, T)}{\bar{V} k T} \frac{\partial \mu(u, v, w, \tau)}{\partial v}\right] \mathrm{d} w \mathrm{~d} v \mathrm{~d} u \mathrm{~d} \tau \frac{2 \pi b_{y}}{\sqrt{D_{0 I} D_{0 V}}}-\frac{2 \pi b_{z}}{\sqrt{D_{0 I} D_{0 V}}} \sum_{n=1}^{\infty} n C_{n}(\chi, \eta, \phi) \\
& \times e_{n I}(\vartheta) \int_{0}^{\vartheta} e_{n I}(-\tau) \int_{0}^{1} \cos (\pi n u) \int_{0}^{1} \cos (\pi n v) \int_{0}^{1} \sin (\pi n w)\left[F_{n I}+\frac{D_{I S}(u, T)}{\bar{V} k T} \frac{\partial \mu(u, v, w, \tau)}{\partial w}\right] \mathrm{d} w \mathrm{~d} v \mathrm{~d} u \mathrm{~d} \tau \text {; } \\
& \tilde{V}_{000}(\chi, \eta, \phi, \vartheta)=-\frac{2 \pi b_{x}}{\sqrt{D_{0 I} D_{0 V}}} \sum_{n=1}^{\infty} n C_{n}(\chi, \eta, \phi) e_{n V}(\vartheta) \int_{0}^{\vartheta} e_{n V}(-\tau) \int_{0}^{1} \sin (\pi n u) \int_{0}^{1} \cos (\pi n v) \int_{0}^{1} \cos (\pi n w)\left[F_{n V}\right. \\
& \left.+\frac{D_{V S}(u, T)}{\bar{V} k T} \frac{\partial \mu(u, v, w, \tau)}{\partial u}\right] \mathrm{d} w \mathrm{~d} v \mathrm{~d} u \mathrm{~d} \tau-\sum_{n=1}^{\infty} n C_{n}(\chi, \eta, \phi) e_{n V}(\vartheta) \int_{0}^{\vartheta} e_{n V}(-\tau) \int_{0}^{1} \cos (\pi n u) \int_{0}^{1} \sin (\pi n v) \\
& \times \int_{0}^{1} \cos (\pi n w)\left[F_{n V}+\frac{D_{V S}(u, T)}{\bar{V} k T} \frac{\partial \mu(u, v, w, \tau)}{\partial v}\right] \mathrm{d} w \mathrm{~d} v \mathrm{~d} u \mathrm{~d} \tau \frac{2 \pi b_{y}}{\sqrt{D_{0 I} D_{0 V}}}-\frac{2 \pi b_{z}}{\sqrt{D_{0 I} D_{0 V}}} \sum_{n=1}^{\infty} n C_{n}(\chi, \eta, \phi) \\
& \times e_{n V}(\vartheta) \int_{0}^{\vartheta} e_{n V}(-\tau) \int_{0}^{1} \cos (\pi n u) \int_{0}^{1} \cos (\pi n v) \int_{0}^{1} \sin (\pi n w)\left[F_{n V}+\frac{D_{V S}(u, T)}{\bar{V} k T} \frac{\partial \mu(u, v, w, \tau)}{\partial w}\right] \mathrm{d} w \mathrm{~d} v \mathrm{~d} u \mathrm{~d} \tau ;
\end{aligned}
$$

where

$C_{n}(\chi, \eta, \phi)=\cos (\pi n \chi) \cos (\pi n \eta) \cos (\pi n \phi) ; F_{n \rho}=\frac{1}{\rho^{*}} \int_{0}^{1} \int_{0}^{1} \int_{0}^{1} C_{n}(u, v, w) f_{n \rho}(u, v, w) \mathrm{d} w \mathrm{~d} v \mathrm{~d} u ;$ $e_{n I}(\vartheta)=\exp \left(-\pi^{2} n^{2} \vartheta \sqrt{D_{0 V} / D_{0 I}}\right) ; e_{n V}(\vartheta)=\exp \left(-\pi^{2} n^{2} \vartheta \sqrt{D_{0 I} / D_{0 V}}\right), c_{n}(\chi)=\cos \left(\pi n \chi / L_{\chi}\right) ;$

$\tilde{I}_{i 00}(\chi, \eta, \phi, \vartheta)=-2 \pi b_{x} \sqrt{\frac{D_{0 I}}{D_{0 V}}} \sum_{n=1}^{\infty} C_{n}(\chi, \eta, \phi) e_{n V}(\vartheta) \int_{0}^{\vartheta} e_{n V}(-\tau) \int_{0}^{1} \sin (\pi n u) \int_{0}^{1} \cos (\pi n v) \int_{0}^{1} \cos (\pi n w) g_{I}(u, T)$ $\times n \frac{\partial \tilde{I}_{i-100}(u, v, w, \tau)}{\partial u} \mathrm{~d} w \mathrm{~d} v \mathrm{~d} u \mathrm{~d} \tau-2 \pi \sum_{n=1}^{\infty} n \int_{0}^{\vartheta} e_{n I}(-\tau) \int_{0}^{1} \cos (\pi n u) g_{I}(u, T) \frac{\partial \tilde{I}_{i-100}(u, v, w, \tau)}{\partial u} \mathrm{~d} w \mathrm{~d} v \mathrm{~d} u \mathrm{~d} \tau$ $\times b_{y} n C_{n}(\chi, \eta, \phi) e_{n I}(\vartheta) \sqrt{\frac{D_{0 I}}{D_{0 V}}}-2 \pi \sqrt{\frac{D_{0 I}}{D_{0 V}}} \sum_{n=1}^{\infty} n C_{n}(\chi, \eta, \phi) e_{n I}(\vartheta) \int_{0}^{\vartheta} e_{n I}(-\tau) \int_{0}^{1} \cos (\pi n u) \int_{0}^{1} \sin (\pi n v) \int_{0}^{1} g_{I}(u, T)$ $\times b_{y} \cos (\pi n w) \frac{\partial \tilde{I}_{i-100}(u, v, w, \tau)}{\partial v} \mathrm{~d} w \mathrm{~d} v \mathrm{~d} u \mathrm{~d} \tau-2 \sqrt{\frac{D_{0 I}}{D_{0 V}}} \sum_{n=1}^{\infty} n C_{n}(\chi, \eta, \phi) \int_{0}^{\vartheta} e_{n I}(-\tau) \int_{0}^{1} \cos (\pi n u) \int_{0}^{1} \cos (\pi n v)$ $\times \int_{0}^{1} \sin (\pi n w) g_{I}(u, v, w, T) \frac{\partial \tilde{I}_{i-100}(u, v, w, \tau)}{\partial w} \mathrm{~d} w \mathrm{~d} v \mathrm{~d} u \mathrm{~d} \tau e_{n I}(\vartheta) \pi b_{z}, i \geq 1 ;$ 
$\tilde{V}_{i 00}(\chi, \eta, \phi, \vartheta)=-2 \pi b_{x} \sqrt{\frac{D_{0 V}}{D_{0 I}}} \sum_{n=1}^{\infty} C_{n}(\chi, \eta, \phi) e_{n V}(\vartheta) \int_{0}^{\vartheta} e_{n V}(-\tau) \int_{0}^{1} \sin (\pi n u) \int_{0}^{1} \cos (\pi n v) \int_{0}^{1} \cos (\pi n w) g_{V}(u, T)$ $\times n \frac{\partial \tilde{V}_{i-100}(u, v, w, \tau)}{\partial u} \mathrm{~d} w \mathrm{~d} v \mathrm{~d} u \mathrm{~d} \tau-2 \pi \sum_{n=1}^{\infty} n \int_{0}^{\vartheta} e_{n V}(-\tau) \int_{0}^{1} \cos (\pi n u) g_{V}(u, T) \frac{\partial \tilde{V}_{i-100}(u, v, w, \tau)}{\partial u} \mathrm{~d} w \mathrm{~d} v \mathrm{~d} u \mathrm{~d} \tau$ $\times b_{y} C_{n}(\chi, \eta, \phi) e_{n V}(\vartheta) \sqrt{\frac{D_{0 V}}{D_{0 I}}}-2 \pi b_{y} \sqrt{\frac{D_{0 V}}{D_{0 I}}} \sum_{n=1}^{\infty} C_{n}(\chi, \eta, \phi) e_{n V}(\vartheta) \int_{0}^{\vartheta} e_{n V}(-\tau) \int_{0}^{1} \cos (\pi n u) \int_{0}^{1} \sin (\pi n v) \int_{0}^{1} g_{V}(u, T)$ $\times n \cos (\pi n w) \frac{\partial \tilde{V}_{i-100}(u, v, w, \tau)}{\partial v} \mathrm{~d} w \mathrm{~d} v \mathrm{~d} u \mathrm{~d} \tau-2 \pi b_{z} \sqrt{\frac{D_{0 V}}{D_{0 I}}} \sum_{n=1}^{\infty} C_{n}(\chi, \eta, \phi) \int_{0}^{\vartheta} e_{n V}(-\tau) \int_{0}^{1} \cos (\pi n u) \int_{0}^{1} \cos (\pi n v)$ $\times n \int_{0}^{1} \sin (\pi n w) g_{V}(u, T) \frac{\partial \tilde{V}_{i-100}(u, v, w, \tau)}{\partial w} \mathrm{~d} w \mathrm{~d} v \mathrm{~d} u \mathrm{~d} \tau e_{n V}(\vartheta), i \geq 1$

$\tilde{\rho}_{010}(\chi, \eta, \phi, \vartheta)=-2 \sum_{n=1}^{\infty} C_{n}(\chi, \eta, \phi) e_{n \rho}(\vartheta) \int_{0}^{\vartheta} e_{n \rho}(-\tau) \int_{0}^{1} \cos (\pi n u) \int_{0}^{1} \cos (\pi n v) \int_{0}^{1} \cos (\pi n w)\left[1+\varepsilon_{I, V} g_{I, V}(u, T)\right]$ $\times \tilde{I}_{000}(u, v, w, \tau) \tilde{V}_{000}(u, v, w, \tau) \mathrm{d} w \mathrm{~d} v \mathrm{~d} u \mathrm{~d} \tau ;$

$\tilde{I}_{020}(\chi, \eta, \phi, \vartheta)=-2 \sum_{n=1}^{\infty} C_{n}(\chi, \eta, \phi) e_{n I}(\vartheta) \int_{0}^{\vartheta} e_{n I}(-\tau) \int_{0}^{1} \cos (\pi n u) \int_{0}^{1} \cos (\pi n v) \int_{0}^{1}\left[1+\varepsilon_{I, V} g_{I, V}(u, T)\right]$ $\times \cos (\pi n w) \sqrt{\frac{D_{0 I}}{D_{0 V}}}\left[\tilde{I}_{010}(u, v, w, \tau) \tilde{V}_{000}(u, v, w, \tau)+\tilde{I}_{000}(u, v, w, \tau) \tilde{V}_{010}(u, v, w, \tau)\right] \mathrm{d} w \mathrm{~d} v \mathrm{~d} u d \tau ;$

$\tilde{V}_{020}(\chi, \eta, \phi, \vartheta)=-2 \sum_{n=1}^{\infty} C_{n}(\chi, \eta, \phi) e_{n V}(\vartheta) \int_{0}^{\vartheta} e_{n V}(-\tau) \int_{0}^{1} \cos (\pi n u) \int_{0}^{1} \cos (\pi n v) \int_{0}^{1}\left[1+\varepsilon_{I, V} g_{I, V}(u, T)\right]$ $\times \cos (\pi n w) \sqrt{\frac{D_{0 V}}{D_{0 I}}}\left[\tilde{I}_{010}(u, v, w, \tau) \tilde{V}_{000}(u, v, w, \tau)+\tilde{I}_{000}(u, v, w, \tau) \tilde{V}_{010}(u, v, w, \tau)\right] \mathrm{d} w \mathrm{~d} v \mathrm{~d} u \mathrm{~d} \tau$

$\tilde{I}_{001}(\chi, \eta, \phi, \vartheta)=-2 \sum_{n=1}^{\infty} C_{n}(\chi, \eta, \phi) e_{n I}(\vartheta) \int_{0}^{\vartheta} e_{n I}(-\tau) \int_{0}^{1} \cos (\pi n u) \int_{0}^{1} \cos (\pi n v) \int_{0}^{1} \cos (\pi n w) \tilde{I}_{000}^{2}(u, v, w, \tau)$ $\times\left[1+\varepsilon_{I, I} g_{I, I}(u, T)\right] \mathrm{d} w \mathrm{~d} v \mathrm{~d} u \mathrm{~d} \tau$

$\tilde{V}_{001}(\chi, \eta, \phi, \vartheta)=-2 \sum_{n=1}^{\infty} C_{n}(\chi, \eta, \phi) e_{n V}(\vartheta) \int_{0}^{\vartheta} e_{n V}(-\tau) \int_{0}^{1} \cos (\pi n u) \int_{0}^{1} \cos (\pi n v) \int_{0}^{1} \cos (\pi n w) \tilde{V}_{000}^{2}(u, v, w, \tau)$ $\times\left[1+\varepsilon_{V, V} g_{V, V}(u, T)\right] \mathrm{d} w \mathrm{~d} v \mathrm{~d} u \mathrm{~d} \tau ;$

$\tilde{I}_{002}(\chi, \eta, \phi, \vartheta)=-2 \sum_{n=1}^{\infty} C_{n}(\chi, \eta, \phi) e_{n I}(\vartheta) \int_{0}^{\vartheta} e_{n I}(-\tau) \int_{0}^{1} \cos (\pi n u) \int_{0}^{1} \cos (\pi n v) \int_{0}^{1} \cos (\pi n w) \tilde{I}_{001}(u, v, w, \tau)$ $\times\left[1+\varepsilon_{I, I} g_{I, I}(u, T)\right] \tilde{I}_{000}(u, v, w, \tau) \mathrm{d} w \mathrm{~d} v \mathrm{~d} u \mathrm{~d} \tau ;$ 
$\tilde{V}_{002}(\chi, \eta, \phi, \vartheta)=-2 \sum_{n=1}^{\infty} C_{n}(\chi, \eta, \phi) e_{n V}(\vartheta) \int_{0}^{\vartheta} e_{n V}(-\tau) \int_{0}^{1} \cos (\pi n u) \int_{0}^{1} \cos (\pi n v) \int_{0}^{1} \cos (\pi n w) \tilde{V}_{001}(u, v, w, \tau)$ $\times\left[1+\varepsilon_{V, V} g_{V, V}(u, T)\right] \tilde{V}_{000}(u, v, w, \tau) \mathrm{d} w \mathrm{~d} v \mathrm{~d} u \mathrm{~d} \tau ;$

$\tilde{I}_{110}(\chi, \eta, \phi, \vartheta)=-2 \pi b_{x} \sqrt{\frac{D_{0 I}}{D_{0 V}}} \sum_{n=1}^{\infty} n C_{n}(\chi, \eta, \phi) e_{n I}(\vartheta) \int_{0}^{\vartheta} e_{n I}(-\tau) \int_{0}^{1} \sin (\pi n u) \int_{0}^{1} \cos (\pi n v) \int_{0}^{1} \cos (\pi n w)$ $\times g_{I}(u, T) \frac{\partial \tilde{I}_{010}(u, v, w, \tau)}{\partial u} \mathrm{~d} w \mathrm{~d} v \mathrm{~d} u \mathrm{~d} \tau-2 \pi b_{y} \sqrt{\frac{D_{0 I}}{D_{0 V}}} \sum_{n=1}^{\infty} n C_{n}(\chi, \eta, \phi) e_{n I}(\vartheta) \int_{0}^{\vartheta} e_{n I}(-\tau) \int_{0}^{1} \cos (\pi n u)$ $\times \int_{0}^{1} \sin (\pi n v) \int_{0}^{1} \cos (\pi n w) g_{I}(u, T) \frac{\partial \tilde{I}_{010}(u, v, w, \tau)}{\partial v} \mathrm{~d} w \mathrm{~d} v \mathrm{~d} u \mathrm{~d} \tau-2 \pi b_{z} \sqrt{\frac{D_{0 I}}{D_{0 V}}} \sum_{n=1}^{\infty} e_{n I}(\vartheta) C_{n}(\chi, \eta, \phi)$ $\times n \int_{0}^{\vartheta} e_{n I}(-\tau) \int_{0}^{1} \cos (\pi n u) \int_{0}^{1} \cos (\pi n v) \int_{0}^{1} \sin (\pi n w) g_{I}(u, T) \frac{\partial \tilde{I}_{010}(u, v, w, \tau)}{\partial w} \mathrm{~d} w \mathrm{~d} v \mathrm{~d} u \mathrm{~d} \tau-2 \sum_{n=1}^{\infty} e_{n I}(\vartheta)$ $\times C_{n}(\chi, \eta, \phi) \int_{0}^{\vartheta} \int_{0}^{1} \cos (\pi n u) \int_{0}^{1} \cos (\pi n v) \int_{0}^{1}\left[\tilde{I}_{100}(u, v, w, \tau) \tilde{V}_{000}(u, v, w, \tau)+\tilde{I}_{000}(u, v, w, \tau) \tilde{V}_{100}(u, v, w, \tau)\right]$ $\times e_{n I}(-\tau)\left[1+\varepsilon_{I, V} g_{I, V}(u, T)\right] \cos (\pi n w) \mathrm{d} w \mathrm{~d} v \mathrm{~d} u \mathrm{~d} \tau ;$

$\tilde{V}_{110}(\chi, \eta, \phi, \vartheta)=-2 \pi b_{x} \sqrt{\frac{D_{0 V}}{D_{0 I}}} \sum_{n=1}^{\infty} n C_{n}(\chi, \eta, \phi) e_{n V}(\vartheta) \int_{0}^{\vartheta} e_{n V}(-\tau) \int_{0}^{1} \sin (\pi n u) \int_{0}^{1} \cos (\pi n v) \int_{0}^{1} \cos (\pi n w)$ $\times g_{V}(u, T) \frac{\partial \tilde{V}_{010}(u, v, w, \tau)}{\partial u} \mathrm{~d} w \mathrm{~d} v \mathrm{~d} u \mathrm{~d} \tau-2 \pi b_{y} \sqrt{\frac{D_{0 V}}{D_{0 I}}} \sum_{n=1}^{\infty} n C_{n}(\chi, \eta, \phi) e_{n V}(\vartheta) \int_{0}^{\vartheta} e_{n V}(-\tau) \int_{0}^{1} \cos (\pi n u)$ $\times \int_{0}^{1} \sin (\pi n v) \int_{0}^{1} \cos (\pi n w) g_{V}(u, T) \frac{\partial \tilde{V}_{010}(u, v, w, \tau)}{\partial v} \mathrm{~d} w \mathrm{~d} v \mathrm{~d} u \mathrm{~d} \tau-2 \pi b_{z} \sqrt{\frac{D_{0 V}}{D_{0 I}}} \sum_{n=1}^{\infty} e_{n V}(\vartheta) C_{n}(\chi, \eta, \phi)$ $\times n \int_{0}^{\vartheta} e_{n V}(-\tau) \int_{0}^{1} \cos (\pi n u) \int_{0}^{1} \cos (\pi n v) \int_{0}^{1} \sin (\pi n w) g_{V}(u, T) \frac{\partial \tilde{V}_{010}(u, v, w, \tau)}{\partial w} \mathrm{~d} w \mathrm{~d} v \mathrm{~d} u \mathrm{~d} \tau-2 \sum_{n=1}^{\infty} e_{n V}(\vartheta)$ $\times C_{n}(\chi, \eta, \phi) \int_{0}^{\vartheta} \int_{0}^{1} \cos (\pi n u) \int_{0}^{1} \cos (\pi n v) \int_{0}^{1}\left[\tilde{I}_{100}(u, v, w, \tau) \tilde{V}_{000}(u, v, w, \tau)+\tilde{I}_{000}(u, v, w, \tau) \tilde{V}_{100}(u, v, w, \tau)\right]$ $\times e_{n V}(-\tau)\left[1+\varepsilon_{I, V} g_{I, V}(u, T)\right] \cos (\pi n w) \mathrm{d} w \mathrm{~d} v \mathrm{~d} u \mathrm{~d} \tau ;$ 


$$
\begin{aligned}
& \tilde{I}_{101}(\chi, \eta, \phi, \vartheta)=-2 \pi b_{x} \sqrt{\frac{D_{0 I}}{D_{0 V}}} \sum_{n=1}^{\infty} C_{n}(\chi, \eta, \phi) e_{n I}(\vartheta) \int_{0}^{\vartheta} e_{n I}(-\tau) \int_{0}^{1} \sin (\pi n u) \int_{0}^{1} \cos (\pi n v) \int_{0}^{1} \cos (\pi n w) g_{I}(u, T) \\
& \times n \frac{\partial \tilde{I}_{001}(u, v, w, \tau)}{\partial u} \mathrm{~d} w \mathrm{~d} v \mathrm{~d} u \mathrm{~d} \tau-2 \pi b_{y} \sqrt{\frac{D_{0 I}}{D_{0 V}}} \sum_{n=1}^{\infty} n C_{n}(\chi, \eta, \phi) e_{n I}(\vartheta) \int_{0}^{\vartheta} e_{n I}(-\tau) \int_{0}^{1} \cos (\pi n u) \int_{0}^{1} \sin (\pi n v) \\
& \times \int_{0}^{1} \cos (\pi n w) g_{I}(u, T) \frac{\partial \tilde{I}_{001}(u, v, w, \tau)}{\partial v} \mathrm{~d} w \mathrm{~d} v \mathrm{~d} u \mathrm{~d} \tau-2 \pi b_{z} \sqrt{\frac{D_{0 I}}{D_{0 V}}} \sum_{n=1}^{\infty} n C_{n}(\chi, \eta, \phi) e_{n I}(\vartheta) \int_{0}^{\vartheta} \int_{0}^{1} \cos (\pi n u) \\
& \times \int_{0}^{1} \cos (\pi n v) \int_{0}^{1} \sin (\pi n w) g_{I}(u, T) \frac{\partial \tilde{I}_{001}(u, v, w, \tau)}{\partial w} \mathrm{~d} w \mathrm{~d} v \mathrm{~d} u e_{n I}(-\tau) d \tau-2 \sum_{n=1}^{\infty} C_{n}(\chi, \eta, \phi) e_{n I}(\vartheta) \int_{0}^{\vartheta} e_{n I}(-\tau) \\
& \times \int_{0}^{1} \cos (\pi n u) \int_{0}^{1} \cos (\pi n v) \int_{0}^{1} \cos (\pi n w) \tilde{I}_{100}(u, v, w, \tau)\left[1+\varepsilon_{I, V} g_{I, V}(u, T)\right] \tilde{V}_{000}(u, v, w, \tau) \mathrm{d} w \mathrm{~d} v \mathrm{~d} u \mathrm{~d} \tau ; \\
& \tilde{V}_{101}(\chi, \eta, \phi, \vartheta)=-2 \pi b_{x} \sqrt{\frac{D_{0 V}}{D_{0 I}}} \sum_{n=1}^{\infty} C_{n}(\chi, \eta, \phi) e_{n V}(\vartheta) \int_{0}^{\vartheta} e_{n V}(-\tau) \int_{0}^{1} \sin (\pi n u) \int_{0}^{1} \cos (\pi n v) \int_{0}^{1} \cos (\pi n w) g_{V}(u, T) \\
& \times n \frac{\partial \tilde{V}_{001}(u, v, w, \tau)}{\partial u} \mathrm{~d} w \mathrm{~d} v \mathrm{~d} u \mathrm{~d} \tau-2 \pi b_{y} \sqrt{\frac{D_{0 V}}{D_{0 I}}} \sum_{n=1}^{\infty} n C_{n}(\chi, \eta, \phi) e_{n V}(\vartheta) \int_{0}^{\vartheta} e_{n V}(-\tau) \int_{0}^{1} \cos (\pi n u) \int_{0}^{1} \sin (\pi n v) \\
& \times \int_{0}^{1} \cos (\pi n w) g_{V}(u, T) \frac{\partial \tilde{V}_{001}(u, v, w, \tau)}{\partial v} \mathrm{~d} w \mathrm{~d} v \mathrm{~d} u \mathrm{~d} \tau-2 \pi b_{z} \sqrt{\frac{D_{0 V}}{D_{0 I}}} \sum_{n=1}^{\infty} n C_{n}(\chi, \eta, \phi) e_{n V}(\vartheta) \int_{0}^{\vartheta} \int_{0}^{1} \cos (\pi n u) \\
& \times \int_{0}^{1} \cos (\pi n v) \int_{0}^{1} \sin (\pi n w) g_{V}(u, T) \frac{\partial \tilde{V}_{001}(u, v, w, \tau)}{\partial w} \mathrm{~d} w \mathrm{~d} v \mathrm{~d} u e_{n V}(-\tau) d \tau-2 \sum_{n=1}^{\infty} C_{n}(\chi, \eta, \phi) e_{n V}(\vartheta) \int_{0}^{\vartheta} e_{n V}(-\tau) \\
& \times \int_{0}^{1} \cos (\pi n u) \int_{0}^{1} \cos (\pi n v) \int_{0}^{1} \cos (\pi n w) \tilde{I}_{000}(u, v, w, \tau)\left[1+\varepsilon_{I, V} g_{I, V}(u, T)\right] \tilde{V}_{100}(u, v, w, \tau) \mathrm{d} w \mathrm{~d} v \mathrm{~d} u \mathrm{~d} \tau ; \\
& \tilde{I}_{011}(\chi, \eta, \phi, \vartheta)=-2 \sum_{n=1}^{\infty} C_{n}(\chi, \eta, \phi) e_{n I}(\vartheta) \int_{0}^{\vartheta} e_{n I}(-\tau) \int_{0}^{1} \cos (\pi n u) \int_{0}^{1} \cos (\pi n v) \int_{0}^{1} \cos (\pi n w)\left\{\tilde{I}_{000}(u, v, w, \tau)[1+\right. \\
& \left.\left.+\varepsilon_{I, I} g_{I, I}(u, v, w, T)\right] \tilde{I}_{010}(u, v, w, \tau)+\left[1+\varepsilon_{I, V} g_{I, V}(u, T)\right] \tilde{I}_{001}(u, v, w, \tau) \tilde{V}_{000}(u, v, w, \tau)\right\} \mathrm{d} w \mathrm{~d} v \mathrm{~d} u \mathrm{~d} \tau ; \\
& \tilde{V}_{011}(\chi, \eta, \phi, \vartheta)=-2 \sum_{n=1}^{\infty} C_{n}(\chi, \eta, \phi) e_{n V}(\vartheta) \int_{0}^{\vartheta} e_{n V}(-\tau) \int_{0}^{1} \cos (\pi n u) \int_{0}^{1} \cos (\pi n v) \int_{0}^{1} \cos (\pi n w)\left\{\tilde{V}_{000}(u, v, w, \tau)[1+\right. \\
& \left.\left.+\varepsilon_{V, V} g_{V, V}(u, v, w, T)\right] \tilde{V}_{010}(u, v, w, \tau)+\left[1+\varepsilon_{I, V} g_{I, V}(u, T)\right] \tilde{I}_{000}(u, v, w, \tau) \tilde{V}_{001}(u, v, w, \tau)\right\} \mathrm{d} w \mathrm{~d} v \mathrm{~d} u \mathrm{~d} \tau ;
\end{aligned}
$$

Equations for functions $\Phi_{\rho i}(x, y, z, t)(i \geq 0)$ and boundary and initial conditions for them could be written as 


$$
\begin{aligned}
& \frac{\partial \Phi_{I 0}(x, y, z, t)}{\partial t}=\frac{\partial}{\partial x}\left[\frac{D_{\Phi_{I} S}(x, T)}{\bar{V} k T} \frac{\partial \mu(x, y, z, t)}{\partial x}\right]+\frac{\partial}{\partial y}\left[\frac{D_{\Phi_{I} S}(x, T)}{\bar{V} k T} \frac{\partial \mu(x, y, z, t)}{\partial y}\right]+\left[\frac{\partial^{2} \Phi_{I 0}(x, y, z, t)}{\partial x^{2}}\right. \\
& \left.+\frac{\partial^{2} \Phi_{I 0}(x, y, z, t)}{\partial y^{2}}+\frac{\partial^{2} \Phi_{I 0}(x, y, z, t)}{\partial z^{2}}\right] D_{0 \Phi I}+\frac{\partial}{\partial z}\left[\frac{D_{\Phi_{I} S}(x, T)}{\bar{V} k T} \frac{\partial \mu(x, y, z, t)}{\partial z}\right]+k_{I, I}(x, T) I^{2}(x, y, z, t) \\
& -k_{I}(x, T) I(x, y, z, t) \\
& \frac{\partial \Phi_{V 0}(x, y, z, t)}{\partial t}=\frac{\partial}{\partial x}\left[\frac{D_{\Phi_{V}}(x, T)}{\bar{V} k T} \frac{\partial \mu(x, y, z, t)}{\partial x}\right]+\frac{\partial}{\partial y}\left[\frac{D_{\Phi_{V}}(x, T)}{\bar{V} k T} \frac{\partial \mu(x, y, z, t)}{\partial y}\right]+\left[\frac{\partial^{2} \Phi_{V 0}(x, y, z, t)}{\partial x^{2}}\right. \\
& \left.+\frac{\partial^{2} \Phi_{V 0}(x, y, z, t)}{\partial y^{2}}+\frac{\partial^{2} \Phi_{V 0}(x, y, z, t)}{\partial z^{2}}\right] D_{0 \Phi V}+\frac{\partial}{\partial z}\left[\frac{D_{\Phi_{V}}(x, T)}{\bar{V} k T} \frac{\partial \mu(x, y, z, t)}{\partial z}\right]+k_{V, V}(x, T) V^{2}(x, y, z, t) \\
& -k_{V}(x, T) V(x, y, z, t) \\
& \frac{\partial \Phi_{I i}(x, y, z, t)}{\partial t}=D_{0 \Phi I}\left\{\frac{\partial}{\partial x}\left[g_{\Phi I}(x, T) \frac{\partial \Phi_{I i-1}(x, y, z, t)}{\partial x}\right]+\frac{\partial}{\partial y}\left[g_{\Phi I}(x, T) \frac{\partial \Phi_{I i-1}(x, y, z, t)}{\partial y}\right]\right. \\
& \left.+\frac{\partial}{\partial z}\left[g_{\Phi I}(x, T) \frac{\partial \Phi_{I i-1}(x, y, z, t)}{\partial z}\right]+\frac{\partial^{2} \Phi_{I i}(x, y, z, t)}{\partial x^{2}}+\frac{\partial^{2} \Phi_{I i}(x, y, z, t)}{\partial y^{2}}+\frac{\partial^{2} \Phi_{I i}(x, y, z, t)}{\partial z^{2}}\right\}, \quad i \geq 1 ; \\
& \frac{\partial \Phi_{V i}(x, y, z, t)}{\partial t}=D_{0 \Phi V}\left\{\frac{\partial}{\partial x}\left[g_{\Phi V}(x, T) \frac{\partial \Phi_{V i-1}(x, y, z, t)}{\partial x}\right]+\frac{\partial}{\partial y}\left[g_{\Phi V}(x, T) \frac{\partial \Phi_{V i-1}(x, y, z, t)}{\partial y}\right]\right. \\
& \left.+\frac{\partial}{\partial z}\left[g_{\Phi V}(x, T) \frac{\partial \Phi_{V i-1}(x, y, z, t)}{\partial z}\right]+\frac{\partial^{2} \Phi_{V i}(x, y, z, t)}{\partial x^{2}}+\frac{\partial^{2} \Phi_{V i}(x, y, z, t)}{\partial y^{2}}+\frac{\partial^{2} \Phi_{V i}(x, y, z, t)}{\partial z^{2}}\right\}, \quad i \geq 1 ; \\
& \left.\frac{\partial \Phi_{\rho i}(x, y, z, t)}{\partial x}\right|_{x=0}=0,\left.\quad \frac{\partial \Phi_{\rho i}(x, y, z, t)}{\partial x}\right|_{x=L_{x}}=0,\left.\quad \frac{\partial \Phi_{\rho i}(x, y, z, t)}{\partial y}\right|_{y=0}=0,\left.\quad \frac{\partial \Phi_{\rho i}(x, y, z, t)}{\partial y}\right|_{y=L_{y}}=0, \\
& \left.\frac{\partial \Phi_{\rho i}(x, y, z, t)}{\partial z}\right|_{z=0}=0,\left.\quad \frac{\partial \Phi_{\rho i}(x, y, z, t)}{\partial z}\right|_{z=L_{z}}=0, \quad i \geq 0
\end{aligned}
$$

$\Phi_{I 0}(x, y, z, 0)=f_{\Phi I}(x, y, z), \Phi_{I i}(x, y, z, 0)=0, \Phi_{V 0}(x, y$,

$z, 0)=f_{\Phi V}(x, y, z), \Phi_{V i}(x, y, z, 0)=0, i \geq 1$.

Solutions of the equations could be written as

$$
\begin{aligned}
& \Phi_{I 0}(x, y, z, t)=-\frac{2 \pi}{L_{x}^{2} L_{y} L_{z}} \sum_{n=1}^{\infty} C_{n}(x, y, z) e_{\Phi I n}(t) \int_{0}^{t} e_{\Phi I n}(-\tau) \int_{0}^{L_{x}} s_{n}(u) \int_{0}^{L_{y}} \int_{0}^{L_{z}}\left[F_{n \Phi_{I}}+\frac{D_{\Phi_{I}}(u, T)}{\bar{V} k T} \frac{\partial \mu(u, v, w, \tau)}{\partial u}\right] \\
& \times n c_{n}(w) d w c_{n}(v) \mathrm{d} v \mathrm{~d} u \mathrm{~d} \tau-\frac{2 \pi}{L_{x} L_{y}^{2} L_{z}} \sum_{n=1}^{\infty} n C_{n}(x, y, z) e_{\Phi I n}(t) \int_{0}^{t} e_{\Phi I n}(-\tau) \int_{0}^{L_{x}} c_{n}(u) \int_{0}^{L_{y}} s_{n}(v) \int_{0}^{L_{z}}\left[\frac{\partial \mu(u, v, w, \tau)}{\partial v}\right. \\
& \left.\times \frac{D_{\Phi_{I} S}(u, T)}{\bar{V} k T}+F_{n \Phi_{I}}\right] c_{n}(w) \mathrm{d} w \mathrm{~d} v \mathrm{~d} u \mathrm{~d} \tau-\pi \frac{2 L_{z}^{-2}}{L_{x} L_{y}} \sum_{n=1}^{\infty} C_{n}(x, y, z) e_{\Phi I n}(t) \int_{0}^{L_{x}} e_{\Phi I n}(-\tau) \int_{0}^{L_{x}} c_{n}(u) \int_{0}^{L_{y}} c_{n}(v) \int_{0}^{L_{z}}\left[F_{n \Phi_{I}}\right. \\
& \left.+\frac{D_{\Phi_{I} S}(u, T)}{\bar{V} k T} \frac{\partial \mu(u, v, w, \tau)}{\partial w}\right] s_{n}(w) \mathrm{d} w \mathrm{~d} v \mathrm{~d} u \mathrm{~d} \tau n+\frac{2}{L_{x} L_{y} L_{z}} \sum_{n=1}^{\infty} C_{n}(x, y, z) e_{\Phi I n}(t) \int_{0}^{t} e_{\Phi I n}(-\tau) \int_{0}^{L_{x}} c_{n}(u) \\
& \quad \times \int_{0}^{L_{y}} c_{n}(v) \int_{0}^{L_{z}} c_{n}(w)\left[k_{I, I}(u, T) I^{2}(u, v, w, \tau)-k_{I}(u, T) I(u, v, w, \tau)\right] \mathrm{d} w \mathrm{~d} v \mathrm{~d} u \mathrm{~d} \tau ;
\end{aligned}
$$


$\Phi_{V 0}(x, y, z, t)=-\frac{2 \pi}{L_{x}^{2} L_{y} L_{z}} \sum_{n=1}^{\infty} C_{n}(x, y, z) e_{\Phi V n}(t) \int_{0}^{t} e_{\Phi V n}(-\tau) \int_{0}^{L_{x}} s_{n}(u) \int_{0}^{L_{y}} \int_{0}^{L_{z}}\left[F_{n \Phi_{V}}+\frac{D_{\Phi_{V} S}(u, T)}{\bar{V} k T} \frac{\partial \mu(u, v, w, \tau)}{\partial u}\right]$

$\times n c_{n}(w) \mathrm{d} w c_{n}(v) \mathrm{d} v \mathrm{~d} u \mathrm{~d} \tau-\frac{2 \pi}{L_{x} L_{y}^{2} L_{z}} \sum_{n=1}^{\infty} n C_{n}(x, y, z) e_{\Phi V n}(t) \int_{0}^{t} e_{\Phi V n}(-\tau) \int_{0}^{L_{x}} c_{n}(u) \int_{0}^{L_{y}} s_{n}(v) \int_{0}^{L_{z}}\left[\frac{\partial \mu(u, v, w, \tau)}{\partial v}\right.$

$\left.\times \frac{D_{\Phi_{V}}(u, T)}{\bar{V} k T}+F_{n \Phi_{V}}\right] c_{n}(w) \mathrm{d} w \mathrm{~d} v \mathrm{~d} u \mathrm{~d} \tau-\pi \frac{2 L_{z}^{-2}}{L_{x} L_{y}} \sum_{n=1}^{\infty} C_{n}(x, y, z) e_{\Phi V n}(t) \int_{0}^{t} e_{\Phi V n}(-\tau) \int_{0}^{L_{x}} c_{n}(u) \int_{0}^{L_{y}} c_{n}(v) \int_{0}^{L_{z}}\left[F_{n \Phi_{V}}\right.$

$\left.+\frac{D_{\Phi_{V}}(u, T)}{\bar{V} k T} \frac{\partial \mu(u, v, w, \tau)}{\partial w}\right] s_{n}(w) \mathrm{d} w \mathrm{~d} v \mathrm{~d} u \mathrm{~d} \tau n+\frac{2}{L_{x} L_{y} L_{z}} \sum_{n=1}^{\infty} C_{n}(x, y, z) e_{\Phi V n}(t) \int_{0}^{t} e_{\Phi V n}(-\tau) \int_{0}^{L_{x}} c_{n}(u)$

$\times \int_{0}^{L_{y}} c_{n}(v) \int_{0}^{L_{z}} c_{n}(w)\left[k_{V, V}(u, T) V^{2}(u, v, w, \tau)-k_{V}(u, T) V(u, v, w, \tau)\right] \mathrm{d} w \mathrm{~d} v \mathrm{~d} u \mathrm{~d} \tau$,

where

$F_{n \Phi_{\rho}}=\int_{0}^{L_{x}} c_{n}(u) \int_{0}^{L_{y}} c_{n}(v) \int_{0}^{L_{z}} c_{n}(w) f_{C}(u, v, w) \mathrm{d} w \mathrm{~d} v \mathrm{~d} u, e_{\Phi \rho n}(t)=\exp \left[-\pi^{2} n^{2} D_{0 \Phi \rho} t\left(\frac{1}{L_{x}^{2}}+\frac{1}{L_{y}^{2}}+\frac{1}{L_{z}^{2}}\right)\right]$,

$C_{n}(x, y, z)=c_{n}(x) c_{n}(y) c_{n}(z)$

$\Phi_{I i}(x, y, z, t)=-\frac{2 \pi}{L_{x}^{2} L_{y} L_{z}} \sum_{n=1}^{\infty} n \int_{0}^{t} e_{\Phi I n}(-\tau) \int_{0}^{L_{x}} s_{n}(u) \int_{0}^{L_{y}} c_{n}(v) \int_{0}^{L_{z}} c_{n}(w) g_{\Phi I}(u, T) \frac{\partial \Phi_{I i-1}(u, v, w, \tau)}{\partial u} \mathrm{~d} w \mathrm{~d} v \mathrm{~d} u \mathrm{~d} \tau$

$\times C_{n}(x, y, z) e_{\Phi I n}(t)-\frac{2 \pi}{L_{x} L_{y}^{2} L_{z}} \sum_{n=1}^{\infty} C_{n}(x, y, z) e_{\Phi I n}(t) \int_{0}^{t} e_{\Phi I n}(-\tau) \int_{0}^{L_{x}} c_{n}(u) \int_{0}^{L_{y}} s_{n}(v) \int_{0}^{L_{z}} g_{\Phi I}(u, T) \frac{\partial \Phi_{I i-1}(u, v, w, \tau)}{\partial v}$

$\times n c_{n}(w) \mathrm{d} w \mathrm{~d} v \mathrm{~d} u \mathrm{~d} \tau-\frac{2 \pi}{L_{x} L_{y} L_{z}^{2}} \sum_{n=1}^{\infty} C_{n}(x, y, z) e_{\Phi I n}(t) \int_{0}^{t} e_{\Phi I n}(-\tau) \int_{0}^{L_{x}} c_{n}(u) \int_{0}^{L_{y}} c_{n}(v) \int_{0}^{L_{z}} s_{n}(w) \frac{\partial \Phi_{I i-1}(u, v, w, \tau)}{\partial w}$

$\times n g_{\Phi I}(u, T) \mathrm{d} w \mathrm{~d} v \mathrm{~d} u \mathrm{~d} \tau, \quad i \geq 1 ;$

$\Phi_{V i}(x, y, z, t)=-\frac{2 \pi}{L_{x}^{2} L_{y} L_{z}} \sum_{n=1}^{\infty} n \int_{0}^{t} e_{\Phi V n}(-\tau) \int_{0}^{L_{x}} s_{n}(u) \int_{0}^{L_{y}} c_{n}(v) \int_{0}^{L_{z}} c_{n}(w) g_{\Phi V}(u, T) \frac{\partial \Phi_{V i-1}(u, v, w, \tau)}{\partial u} \mathrm{~d} w \mathrm{~d} v \mathrm{~d} u \mathrm{~d} \tau$

$\times C_{n}(x, y, z) e_{\Phi V n}(t)-\frac{2 \pi}{L_{x} L_{y}^{2} L_{z}} \sum_{n=1}^{\infty} C_{n}(x, y, z) e_{\Phi V n}(t) \int_{0}^{t} e_{\Phi V n}(-\tau) \int_{0}^{L_{x}} c_{n}(u) \int_{0}^{L_{y}} s_{n}(v) \int_{0}^{L_{z}} g_{\Phi V}(u, T) \frac{\partial \Phi_{V i-1}(u, v, w, \tau)}{\partial v}$

$\times n c_{n}(w) \mathrm{d} w \mathrm{~d} v \mathrm{~d} u \mathrm{~d} \tau-\frac{2 \pi}{L_{x} L_{y} L_{z}^{2}} \sum_{n=1}^{\infty} C_{n}(x, y, z) e_{\Phi V n}(t) \int_{0}^{t} e_{\Phi V n}(-\tau) \int_{0}^{L_{x}} c_{n}(u) \int_{0}^{L_{y}} c_{n}(v) \int_{0}^{L_{z}} s_{n}(w) \frac{\partial \Phi_{V i-1}(u, v, w, \tau)}{\partial w}$

$\times n g_{\Phi V}(u, T) \mathrm{d} w \mathrm{~d} v \mathrm{~d} u \mathrm{~d} \tau, \quad i \geq 1$.

Equations for the functions $C_{i j}(x, y, z, t)(i \geq 0, j \geq 0)$,

boundary and initial conditions for them are 


$$
\begin{aligned}
& \frac{\partial C_{00}(x, y, z, t)}{\partial t}=D_{0 L} \frac{\partial^{2} C_{00}(x, y, z, t)}{\partial x^{2}}+D_{0 L} \frac{\partial^{2} C_{00}(x, y, z, t)}{\partial y^{2}}+D_{0 L} \frac{\partial^{2} C_{00}(x, y, z, t)}{\partial z^{2}} \\
& +\frac{\partial}{\partial x}\left[\frac{D_{C S}}{\bar{V} k T} \frac{\partial \mu(x, y, z, t)}{\partial x}\right]+\frac{\partial}{\partial y}\left[\frac{D_{C S}}{\bar{V} k T} \frac{\partial \mu(x, y, z, t)}{\partial y}\right]+\frac{\partial}{\partial z}\left[\frac{D_{C S}}{\bar{V} k T} \frac{\partial \mu(x, y, z, t)}{\partial z}\right] ; \\
& \frac{\partial C_{i 0}(x, y, z, t)}{\partial t}=D_{0 L} \frac{\partial^{2} C_{i 0}(x, y, z, t)}{\partial x^{2}}+D_{0 L} \frac{\partial^{2} C_{i 0}(x, y, z, t)}{\partial y^{2}}+D_{0 L} \frac{\partial}{\partial x}\left[g_{L}(x, T) \frac{\partial C_{i-10}(x, y, z, t)}{\partial x}\right] \\
& +D_{0 L} \frac{\partial^{2} C_{i 0}(x, y, z, t)}{\partial z^{2}}+D_{0 L} \frac{\partial}{\partial y}\left[g_{L}(x, T) \frac{\partial C_{i-10}(x, y, z, t)}{\partial y}\right]+D_{0 L} \frac{\partial}{\partial z}\left[g_{L}(x, T) \frac{\partial C_{i-10}(x, y, z, t)}{\partial z}\right], \quad i \geq 1 ; \\
& \frac{\partial C_{01}(x, y, z, t)}{\partial t}=D_{0 L} \frac{\partial^{2} C_{01}(x, y, z, t)}{\partial x^{2}}+D_{0 L} \frac{\partial^{2} C_{01}(x, y, z, t)}{\partial y^{2}}+D_{0 L} \frac{\partial}{\partial x}\left[\frac{C_{00}^{\gamma}(x, y, z, t)}{P^{\gamma}(x, T)} \frac{\partial C_{00}(x, y, z, t)}{\partial x}\right] \\
& +D_{0 L} \frac{\partial^{2} C_{01}(x, y, z, t)}{\partial z^{2}}+D_{0 L} \frac{\partial}{\partial y}\left[\frac{C_{00}^{\gamma}(x, y, z, t)}{P^{\gamma}(x, T)} \frac{\partial C_{00}(x, y, z, t)}{\partial y}\right]+D_{0 L} \frac{\partial}{\partial z}\left[\frac{C_{00}^{\gamma}(x, y, z, t)}{P^{\gamma}(x, T)} \frac{\partial C_{00}(x, y, z, t)}{\partial z}\right] ; \\
& \frac{\partial C_{02}(x, y, z, t)}{\partial t}=\left\{\frac{\partial}{\partial x}\left[C_{01}(x, y, z, t) \frac{C_{00}^{\gamma-1}(x, y, z, t)}{P^{\gamma}(x, T)} \frac{\partial C_{00}(x, y, z, t)}{\partial x}\right]+\frac{\partial}{\partial y}\left[C_{01}(x, y, z, t) \frac{C_{00}^{\gamma-1}(x, y, z, t)}{P^{\gamma}(x, T)}\right.\right. \\
& \left.\times \frac{\partial C_{00}(x, y, z, t)}{\partial y}\right]+\frac{\partial}{\partial z}\left[C_{01}(x, y, z, t) \frac{C_{00}^{\gamma-1}(x, y, z, t)}{P \gamma(x, T)} \frac{\partial C_{00}(x, y, z, t)}{\partial x}\right]+\frac{\partial}{\partial x}\left[\frac{C_{00}^{\gamma}(x, y, z, t)}{P^{\gamma}(x, T)} \frac{\partial C_{01}(x, y, z, t)}{\partial x}\right] \\
& +\frac{\partial}{\partial y}\left[\frac{C_{00}^{\gamma}(x, y, z, t)}{P^{\gamma}(x, T)} \frac{\partial C_{01}(x, y, z, t)}{\partial y}\right]+\frac{\partial}{\partial z}\left[\frac{C_{00}^{\gamma}(x, y, z, t)}{P^{\gamma}(x, t)} \frac{\partial C_{01}(x, y, z, t)}{\partial z}\right]+\frac{\partial^{2} C_{02}(x, y, z, t)}{\partial x^{2}} \\
& \left.+\frac{\partial^{2} C_{02}(x, y, z, t)}{\partial y^{2}}+\frac{\partial^{2} C_{02}(x, y, z, t)}{\partial z^{2}}\right\} D_{0 L} \\
& \frac{\partial C_{11}(x, y, z, t)}{\partial t}=\left\{\frac{\partial}{\partial x}\left[g_{L}(x, T) \frac{\partial C_{01}(x, y, z, t)}{\partial x}\right]+\frac{\partial}{\partial y}\left[g_{L}(x, T) \frac{\partial C_{01}(x, y, z, t)}{\partial y}\right]+\frac{\partial}{\partial z}\left[g_{L}(x, T)\right.\right. \\
& \left.\times \frac{\partial C_{01}(x, y, z, t)}{\partial z}\right]+\frac{\partial}{\partial x}\left[\frac{C_{00}^{\gamma}(x, y, z, t)}{P^{\gamma}(x, T)} \frac{\partial C_{10}(x, y, z, t)}{\partial x}\right]+\frac{\partial}{\partial y}\left[\frac{C_{00}^{\gamma}(x, y, z, t)}{P^{\gamma}(x, T)} \frac{\partial C_{10}(x, y, z, t)}{\partial y}\right] \\
& +\frac{\partial}{\partial z}\left[\frac{C_{00}^{\gamma}(x, y, z, t)}{P^{\gamma}(x, T)} \frac{\partial C_{10}(x, y, z, t)}{\partial z}\right]+\frac{\partial}{\partial x}\left[C_{10}(x, y, z, t) \frac{C_{00}^{\gamma-1}(x, y, z, t)}{P^{\gamma}(x, T)} \frac{\partial C_{00}(x, y, z, t)}{\partial x}\right] \\
& +\frac{\partial}{\partial y}\left[C_{10}(x, y, z, t) \frac{C_{00}^{\gamma-1}(x, y, z, t)}{P^{\gamma}(x, T)} \frac{\partial C_{00}(x, y, z, t)}{\partial y}\right]+\frac{\partial}{\partial z}\left[C_{10}(x, y, z, t) \frac{C_{00}^{\gamma-1}(x, y, z, t)}{P^{\gamma}(x, T)} \frac{\partial C_{00}(x, y, z, t)}{\partial z}\right] \\
& \left.+\frac{\partial^{2} C_{11}(x, y, z, t)}{\partial x^{2}}+\frac{\partial^{2} C_{11}(x, y, z, t)}{\partial y^{2}}+\frac{\partial^{2} C_{11}(x, y, z, t)}{\partial z^{2}}\right\} D_{0 L} \\
& \left.\frac{\partial C_{i j}(x, y, z, t)}{\partial x}\right|_{x=0}=0,\left.\quad \frac{\partial C_{i j}(x, y, z, t)}{\partial x}\right|_{x=L_{x}}=0,\left.\quad \frac{\partial C_{i j}(x, y, z, t)}{\partial y}\right|_{y=0}=0,\left.\quad \frac{\partial C_{i j}(x, y, z, t)}{\partial y}\right|_{y=L_{y}}=0, \\
& \left.\frac{\partial C_{i j}(x, y, z, t)}{\partial z}\right|_{z=0}=0,\left.\quad \frac{\partial C_{i j}(x, y, z, t)}{\partial z}\right|_{z=L_{z}}=0 \\
& i \geq 0, j \geq 0 ; C_{00}(x, y, z, 0)=f_{C}(x, y, z), C_{i j}(x, y, z, 0)=0, i \geq 1, j \geq 1 \text {. }
\end{aligned}
$$




$$
\begin{aligned}
& C_{00}(x, y, z, t)=-\frac{2 \pi}{L_{x}^{2} L_{y} L_{z}} \sum_{n=1}^{\infty}\left[F_{n C}+\int_{0}^{t} e_{n C}(-\tau) \int_{0}^{L_{x}} s_{n}(u) \int_{0}^{L_{y}} c_{n}(v) \int_{0}^{L_{z}} c_{n}(w) \frac{D_{\mathrm{CS}}}{\bar{V} k T} \frac{\partial \mu(u, v, w, \tau)}{\partial u} \mathrm{~d} w \mathrm{~d} v \mathrm{~d} u \mathrm{~d} \tau\right] \\
& \times n C_{n}(x, y, z) e_{n C}(t)-\frac{2 \pi}{L_{x} L_{y}^{2} L_{z}} \sum_{n=1}^{\infty} n\left[\int_{0}^{t} e_{n C}(-\tau) \int_{0}^{L_{x}} c_{n}(u) \int_{0}^{L_{y}} s_{n}(v) \int_{0}^{L_{z}} c_{n}(w) \frac{D_{\mathrm{CS}}}{\bar{V} k T} \frac{\partial \mu(u, v, w, \tau)}{\partial v} \mathrm{~d} w \mathrm{~d} v \mathrm{~d} u \mathrm{~d} \tau\right. \\
& \left.+F_{n C}\right] C_{n}(x, y, z) e_{n C}(t)-\frac{2 \pi}{L_{x} L_{y} L_{z}^{2}} \sum_{n=1}^{\infty}\left[\int_{0}^{t} e_{n C}(-\tau) \int_{0}^{L_{x}} c_{n}(u) \int_{0}^{L_{y}} c_{n}(v) \int_{0}^{L_{z}} s_{n}(w) \frac{D_{\mathrm{CS}}}{\bar{V} k T} \frac{\partial(u, v, w, \tau)}{\partial w} \mathrm{~d} w \mathrm{~d} v \mathrm{~d} u \mathrm{~d} \tau\right. \\
& \left.+F_{n C}\right] n C_{n}(x, y, z) e_{n C}(t),
\end{aligned}
$$

where

$F_{n C}=\int_{0}^{L_{x}} c_{n}(u) \int_{0}^{L_{y}} c_{n}(v) \int_{0}^{L_{z}} c_{n}(w) f_{C}(u, v, w) \mathrm{d} w \mathrm{~d} v \mathrm{~d} u, e_{n C}(t)=\exp \left[-\pi^{2} n^{2} D_{0 L}\left(\frac{1}{L_{x}^{2}}+\frac{1}{L_{y}^{2}}+\frac{1}{L_{z}^{2}}\right)\right]$

$C_{i 0}(x, y, z, t)=-\frac{2 \pi}{L_{x}^{2} L_{y} L_{z}} \sum_{n=1}^{\infty} n F_{n C} C_{n}(x, y, z) e_{n C}(t) \int_{0}^{t} e_{n C}(-\tau) \int_{0}^{L_{x}} s_{n}(u) \int_{0}^{L_{y}} c_{n}(v) \int_{0}^{L_{z}} g_{L}(u, T) \frac{\partial C_{i-10}(u, v, w, \tau)}{\partial u}$

$\times c_{n}(w) \mathrm{d} w \mathrm{~d} v \mathrm{~d} u \mathrm{~d} \tau-\frac{2 \pi}{L_{x} L_{y}^{2} L_{z}} \sum_{n=1}^{\infty} n C_{n}(x, y, z) e_{n C}(t) \int_{0}^{t} e_{n C}(-\tau) \int_{0}^{L_{x}} c_{n}(u) \int_{0}^{L_{y}} s_{n}(v) \int_{0}^{L_{z}} c_{n}(w) \frac{\partial C_{i-10}(u, v, w, \tau)}{\partial v}$

$\times F_{n C} g_{L}(u, T) \mathrm{d} w \mathrm{~d} v \mathrm{~d} u \mathrm{~d} \tau-\frac{2 \pi}{L_{x} L_{y} L_{z}^{2}} \sum_{n=1}^{\infty} n C_{n}(x, y, z) e_{n C}(t) \int_{0}^{t} e_{n C}(-\tau) \int_{0}^{L_{x}} c_{n}(u) \int_{0}^{L_{y}} c_{n}(v) \int_{0}^{L_{z}} \frac{\partial C_{i-10}(u, v, w, \tau)}{\partial w}$

$\times F_{n C} s_{n}(w) g_{L}(u, T) \mathrm{d} w \mathrm{~d} v \mathrm{~d} u \mathrm{~d} \tau, \quad i \geq 1 ;$

$C_{01}(x, y, z, t)=-\frac{2 \pi}{L_{x}^{2} L_{y} L_{z}} \sum_{n=1}^{\infty} n F_{n C} C_{n}(x, y, z) e_{n C}(t) \int_{0}^{t} e_{n C}(-\tau) \int_{0}^{L_{x}} s_{n}(u) \int_{0}^{L_{y}} c_{n}(v) \int_{0}^{L_{z}} c_{n}(w) \frac{C_{00}^{\gamma}(u, v, w, \tau)}{P^{\gamma}(u, T)}$

$\times \frac{\partial C_{00}(u, v, w, \tau)}{\partial u} \mathrm{~d} w \mathrm{~d} v \mathrm{~d} u \mathrm{~d} \tau-\frac{2 \pi}{L_{x} L_{y}^{2} L_{z}} \sum_{n=1}^{\infty} n F_{n C} C_{n}(x, y, z) e_{n C}(t) \int_{0}^{t} e_{n C}(-\tau) \int_{0}^{L_{x}} c_{n}(u) \int_{0}^{L_{y}} s_{n}(v) \int_{0}^{L_{z}} c_{n}(w)$

$\times \frac{C_{00}^{\gamma}(u, v, w, \tau)}{P^{\gamma}(u, T)} \frac{\partial C_{00}(u, v, w, \tau)}{\partial v} \mathrm{~d} w \mathrm{~d} v \mathrm{~d} u \mathrm{~d} \tau-\frac{2 \pi}{L_{x} L_{y} L_{z}^{2}} \sum_{n=1}^{\infty} n F_{n C} C_{n}(x, y, z) e_{n C}(t) \int_{0}^{t} e_{n C}(-\tau) \int_{0}^{L_{x}} c_{n}(u)$

$\times \int_{0}^{L_{y}} c_{n}(v) \int_{0}^{L_{z}} s_{n}(w) \frac{C_{00}^{\gamma}(u, v, w, \tau)}{P \gamma(u, T)} \frac{\partial C_{00}(u, v, w, \tau)}{\partial w} \mathrm{~d} w \mathrm{~d} v \mathrm{~d} u \mathrm{~d} \tau$ 


$$
\begin{aligned}
& C_{02}(x, y, z, t)=-\frac{2 \pi}{L_{x}^{2} L_{y} L_{z}} \sum_{n=1}^{\infty} C_{n}(x, y, z) e_{n C}(t) \int_{0}^{t} e_{n C}(-\tau) \int_{0}^{L_{x}} s_{n}(u) \int_{0}^{L_{y}} c_{n}(v) \int_{0}^{L_{z}} c_{n}(w) C_{01}(u, v, w, \tau) \frac{C_{00}^{\gamma-1}(u, v, w, \tau)}{P^{\gamma}(u, T)} \\
& \times \frac{\partial C_{00}(u, v, w, \tau)}{\partial u} \mathrm{~d} w \mathrm{~d} v \mathrm{~d} u \mathrm{~d} \tau n F_{n C}-\frac{2 \pi}{L_{x} L_{y}^{2} L_{z}} \sum_{n=1}^{\infty} n F_{n C} C_{n}(x, y, z) e_{n C}(t) \int_{0}^{t} e_{n C}(-\tau) \int_{0}^{L_{x}} c_{n}(u) \int_{0}^{L_{y}} s_{n}(v) \int_{0}^{L_{z}} c_{n}(w) \\
& \times C_{01}(u, v, w, \tau) \frac{C_{00}^{\gamma-1}(u, v, w, \tau)}{P^{\gamma}(u, T)} \frac{\partial C_{00}(u, v, w, \tau)}{\partial v} \mathrm{~d} w \mathrm{~d} v \mathrm{~d} u \mathrm{~d} \tau-\frac{2 \pi}{L_{x} L_{y} L_{z}^{2}} \sum_{n=1}^{\infty} F_{n C} C_{n}(x, y, z) e_{n C}(t) \int_{0}^{t} e_{n C}(-\tau) \\
& \times n \int_{0}^{L_{x}} c_{n}(u) \int_{0}^{L_{y}} c_{n}(v) \int_{0}^{L_{z}} s_{n}(w) C_{01}(u, v, w, \tau) \frac{C_{00}^{\gamma-1}(u, v, w, \tau)}{P^{\gamma}(u, T)} \frac{\partial C_{00}(u, v, w, \tau)}{\partial w} \mathrm{~d} w \mathrm{~d} v \mathrm{~d} u \mathrm{~d} \tau-\frac{2 \pi}{L_{x}^{2} L_{y} L_{z}} \sum_{n=1}^{\infty} n F_{n C} \\
& \times C_{n}(x, y, z) e_{n C}(t) \int_{0}^{t} e_{n C}(-\tau) \int_{0}^{L_{x}} s_{n}(u) \int_{0}^{L_{y}} c_{n}(v) \int_{0}^{L_{z}} c_{n}(w) \frac{C_{00}^{\gamma}(u, v, w, \tau)}{P^{\gamma}(u, T)} \frac{\partial C_{01}(u, v, w, \tau)}{\partial u} \mathrm{~d} w \mathrm{~d} v \mathrm{~d} u \mathrm{~d} \tau-\frac{2 \pi}{L_{x} L_{y}^{2} L_{z}} \\
& \times \sum_{n=1}^{\infty} n F_{n C} C_{n}(x, y, z) e_{n C}(t) \int_{0}^{t} e_{n C}(-\tau) \int_{0}^{L_{x}} s_{n}(u) \int_{0}^{L_{y}} c_{n}(v) \int_{0}^{L_{z}} c_{n}(w) \frac{C_{00}^{\gamma}(u, v, w, \tau)}{P^{\gamma}(u, T)} \frac{\partial C_{01}(u, v, w, \tau)}{\partial v} \mathrm{~d} w \mathrm{~d} v \mathrm{~d} u \mathrm{~d} \tau \\
& -2 \sum_{n=1}^{\infty} \frac{\pi n F_{n C}}{L_{x} L_{y} L_{z}^{2}} C_{n}(x, y, z) e_{n C}(t) \int_{0}^{t} e_{n C}(-\tau) \int_{0}^{L_{x}} s_{n}(u) \int_{0}^{L_{y}} c_{n}(v) \int_{0}^{L_{z}} c_{n}(w) \frac{C_{00}^{\gamma}(u, v, w, \tau)}{P^{\gamma}(u, T)} \frac{\partial C_{01}(u, v, w, \tau)}{\partial w} \mathrm{~d} w \mathrm{~d} v \mathrm{~d} u \mathrm{~d} \tau ;
\end{aligned}
$$

$C_{11}(x, y, z, t)=-\frac{2 \pi}{L_{x}^{2} L_{y} L_{z}} \sum_{n=1}^{\infty} e_{n C}(t) \int_{0}^{t} e_{n C}(-\tau) \int_{0}^{L_{x}} s_{n}(u) \int_{0}^{L_{y}} c_{n}(v) \int_{0}^{L_{z}} g_{L}(u, T) \frac{\partial C_{01}(u, v, w, \tau)}{\partial u} c_{n}(w) \mathrm{d} w \mathrm{~d} v \mathrm{~d} u \mathrm{~d} \tau$ $\times n F_{n C} C_{n}(x, y, z)-\frac{2 \pi}{L_{x} L_{y}^{2} L_{z}} \sum_{n=1}^{\infty} F_{n C} \int_{0}^{t} e_{n C}(-\tau) \int_{0}^{L_{x}} c_{n}(u) \int_{0}^{L_{y}} s_{n}(v) \int_{0}^{L_{z}} c_{n}(w) g_{L}(u, T) \frac{\partial C_{01}(u, v, w, \tau)}{\partial v} \mathrm{~d} w \mathrm{~d} v \mathrm{~d} u \mathrm{~d} \tau$ $\times n C_{n}(x, y, z) e_{n C}(t)-\frac{2 \pi}{L_{x} L_{y} L_{z}^{2}} \sum_{n=1}^{\infty} F_{n C} C_{n}(x, y, z) e_{n C}(t) \int_{0}^{t} e_{n C}(-\tau) \int_{0}^{L_{x}} c_{n}(u) \int_{0}^{L_{y}} c_{n}(v) \int_{0}^{L_{z}} g_{L}(u, T) \frac{\partial C_{01}(u, v, w, \tau)}{\partial w}$ $\times n s_{n}(w) \mathrm{d} w \mathrm{~d} v \mathrm{~d} u \mathrm{~d} \tau-\frac{2 \pi}{L_{x}^{2} L_{y} L_{z}} \sum_{n=1}^{\infty} n C_{n}(x, y, z) e_{n C}(t) \int_{0}^{t} e_{n C}(-\tau) \int_{0}^{L_{x}} s_{n}(u) \int_{0}^{L_{y}} c_{n}(v) \int_{0}^{L_{z}} c_{n}(w) \frac{\partial C_{10}(u, v, w, \tau)}{\partial u}$ $\times F_{n C} \frac{C_{00}^{\gamma}(u, v, w, \tau)}{P_{\gamma}^{\gamma}(u, T)} \mathrm{d} w \mathrm{~d} v \mathrm{~d} u \mathrm{~d} \tau-\frac{2 \pi}{L_{x} L_{y}^{2} L_{z}} \sum_{n=1}^{\infty} n e_{n C}(t) \int_{0}^{t} e_{n C}(-\tau) \int_{0}^{L_{x}} c_{n}(u) \int_{0}^{L_{y}} s_{n}(v) \int_{0}^{L_{z}} c_{n}(w) \frac{\partial C_{10}(u, v, w, \tau)}{\partial v}$ $\times n F_{n C} \frac{C_{00}^{\gamma}(u, v, w, \tau)}{P^{\gamma}(u, T)} \mathrm{d} w \mathrm{~d} v \mathrm{~d} u \mathrm{~d} \tau C_{n}(x, y, z)-\frac{2 \pi}{L_{x} L_{y} L_{z}^{2}} \sum_{n=1}^{\infty} F_{n C} C_{n}(x, y, z) e_{n C}(t) \int_{0}^{t} e_{n C}(-\tau) \int_{0}^{L_{x}} c_{n}(u) \int_{0}^{L_{y}} c_{n}(v)$ $\times n \int_{0}^{L_{z}} s c_{n}(w) \frac{C_{00}^{\gamma}(u, v, w, \tau)}{P^{\gamma}(u, T)} \frac{\partial C_{10}(u, v, w, \tau)}{\partial w} \mathrm{~d} w \mathrm{~d} v \mathrm{~d} u \mathrm{~d} \tau-\frac{2 \pi}{L_{x}^{2} L_{y} L_{z}} \sum_{n=1}^{\infty} n F_{n C} C_{n}(x, y, z) e_{n C}(t) \int_{0}^{t} e_{n C}(-\tau)$ $\times \int_{0}^{L_{x}} s_{n}(u) \int_{0}^{L_{y}} c_{n}(v) \int_{0}^{L_{z}} c_{n}(w) C_{10}(u, v, w, \tau) \frac{C_{00}^{\gamma-1}(u, v, w, \tau)}{P^{\gamma}(u, T)} \frac{\partial C_{00}(u, v, w, \tau)}{\partial u} \mathrm{~d} w \mathrm{~d} v \mathrm{~d} u \mathrm{~d} \tau-\frac{2 \pi}{L_{x}^{2} L_{y} L_{z}} \sum_{n=1}^{\infty} n e_{n C}(t)$ 


$$
\begin{aligned}
& \times C_{n}(x, y, z) \int_{0}^{t} e_{n C}(-\tau) \int_{0}^{L_{x}} s_{n}(u) \int_{0}^{L_{y}} c_{n}(v) \int_{0}^{L_{z}} c_{n}(w) C_{10}(u, v, w, \tau) \frac{C_{00}^{\gamma-1}(u, v, w, \tau)}{P \gamma(u, T)} \frac{\partial C_{00}(u, v, w, \tau)}{\partial u} \mathrm{~d} w \mathrm{~d} v \mathrm{~d} u \mathrm{~d} \tau \\
& \times F_{n C}-\frac{2 \pi}{L_{x} L_{y}^{2} L_{z}} \sum_{n=1}^{\infty} C_{n}(x, y, z) e_{n C}(t) \int_{0}^{L_{x}} e_{n C}(-\tau) \int_{0}^{L_{x}} s_{n}(u) \int_{0}^{L_{y}} c_{n}(v) \int_{0}^{L_{z}} C_{10}(u, v, w, \tau) \frac{C_{00}^{\gamma-1}(u, v, w, \tau)}{P \gamma(u, T)} \frac{\partial C_{00}(u, v, w, \tau)}{\partial v} \\
& \times c_{n}(w) \mathrm{d} w \mathrm{~d} v \mathrm{~d} u \mathrm{~d} \tau n F_{n C}-\frac{2 \pi}{L_{x} L_{y} L_{z}^{2}} \sum_{n=1}^{\infty} n C_{n}(x, y, z) e_{n C}(t) \int_{0}^{L_{x}} e_{n C}(-\tau) \int_{0}^{L_{y}} s_{n}(u) \int_{0}^{L_{y}} c_{n}(v) \int_{0}^{L_{z}} c_{n}(w) C_{10}(u, v, w, \tau) \\
& \times F_{n C} \frac{C_{00}^{\gamma-1}(u, v, w, \tau)}{P \gamma(u, T)} \frac{\partial C_{00}(u, v, w, \tau)}{\partial u} \mathrm{~d} w \mathrm{~d} v \mathrm{~d} u \mathrm{~d} \tau
\end{aligned}
$$

\section{References}

Ahlgren T, Likonen J, Slotte J, Räisänen J, Rajatore M, Keinonen J (1997) Concentration dependent and independent $S i$ diffusion in ion-implanted GaAs. Phys Rev B 56(8):4597

Bykov YuV, Yeremeev AG, Zharova NA, Plotnikov IV, Rybakov KI, Drozdov MN, Drozdov YuN, Skupov VD (2003) Diffusion processes in semiconductor structures during microwave annealing. Radiophys Quantum Electron 43(3):836

Cheremskoy PG, Slesov VV, Betekhtin VI (1990) Pore in solid bodies. Energoatomizdat, Moscow (in Russian)

Fahey PM, Griffin PB, Plummer JD (1989) Point defects and dopant diffusion in silicon. Rev Mod Phys 61:289

Gotra ZY (1991) Technology of microelectronic devices. Radio and communication, Moscow

Grebene AB (1983) Bipolar and MOS analogous integrated circuit design. Wiley, New York

Kitayama M, Narushima T, Carter WC, Cannon RM, Glaeser AM (2000a) The Wulff shape of alumina: I. Modeling the kinetics of morphological evolution. J Am Ceram Soc 83:2561

Kitayama M, Narushima T, Glaeser AM (2000b) The Wulff shape of Alumina: II, experimental measurements of pore shape evolution rates. J Am Ceram Soc 83:2572

Lachin VI, Savelov NS (2001) Electronics. Phoenix, Rostov-na-Donu (in Russian)

Mynbaeva MG, Mokhov EN, Lavrent'ev AA, Mynbaev KD (2008) About hightemperature diffusion doping of porous SiC. Techn Phys Lett 34(17):13

Noda T (2003) Indium segregation to dislocation loops induced by ion implantation damage in silicon. J Appl Phys 93(3):1428

Ong KK, Pey KL, Lee PS, Wee ATS, Wang XC, Chong YF (2006) Dopant distribution in the recrystallization transient at the maximum melt depth induced by laser annealing. Appl Phys Lett 89(17):172111
Pankratov EL (2005) Influence of spatial, temporal and concentrational dependence of diffusion coefficient on dopant dynamics: optimization of annealing time. Phys Rev B 72(7):075201

Pankratov EL (2007) Dopant diffusion dynamics and optimal diffusion time as influenced by diffusion-coefficient nonuniformity. Russ Microelectron 36(1):33

Pankratov EL (2008a) Redistribution of dopant, implanted in a multilayer structure for production of a $p$ - $n$-junction, during annealing radiative defects. Phys Lett A 372(11):1897

Pankratov EL (2008b) Analysis of redistribution of radiation defects with account diffusion and several secondary processes. Mod Phys Lett B 22(28):2779

Pankratov EL (2010a) Optimization of near-surficial annealing for decreasing of depth of $p$ - $n$-junction in semiconductor heterostructure. Proc SPIE 7521:75211D

Pankratov EL (2010b) Influence of porosity of materials on redistribution of dopant during manufacturing a diffusion-junction rectifiers in semiconductor heterostructures. Mod Phy Lett B. 24(32):3049-3069

Pankratov EL (2011) Application of porous layers and optimization of annealing of dopant and radiation defects to increase sharpness of $p$ - $n$-junctions in a bipolar heterotransistors. J Nanoel Optoel 6(2): 194

Pankratov EL, Bulaeva EA (2011) Decreasing of quantity of radiation defects in an implanted-junction rectifier in a semiconductor heterostructure. Int J Micro Nano Scale Transp 2(1):85

Pankratov EL, Spagnolo B (2005) Optimization of impurity profile for $p$ - $n$-junction in heterostructures. Eur Phys J B 46(1):15

Tikhonov AN, Samarskii AA (1972) The mathematical physics equations. Nauka, Moscow (in Russian)

Vinetsky VL, Kholodar' GA (1979) Radiation physics in semiconductors. Naukova Dumka, Kiev

Zorin EI, Pavlov PV, Tetelbaum DI (1975) Ion doping of semiconductors. Energiya, Moscow (in Russian) 